\title{
Fixed point problems and a system of generalized nonlinear mixed variational inequalities
}

\author{
Narin Petrot ${ }^{1,2}$ and Javad Balooee $3^{3^{*}}$
}

\section{"Correspondence:}

javad.balooee@gmail.com

${ }^{3}$ Department of Mathematics, Sari

Branch, Islamic Azad University, Sari,

Iran

Full list of author information is

available at the end of the article

\section{Springer}

\begin{abstract}
In this paper, we introduce and consider a new system of generalized nonlinear mixed variational inequalities involving six different nonlinear operators and discuss the existence and uniqueness of solution of the aforesaid system. We use three nearly uniformly Lipschitzian mappings $S_{i}(i=1,2,3)$ to suggest and analyze some new three-step resolvent iterative algorithms with mixed errors for finding an element of the set of fixed points of the nearly uniformly Lipschitzian mapping $\mathcal{Q}=\left(S_{1}, S_{2}, S_{3}\right)$, which is the unique solution of the system of generalized nonlinear mixed variational inequalities. The convergence analysis of the suggested iterative algorithms under suitable conditions is studied. In the final section, an important remark on a class of some relaxed cocoercive mappings is discussed.

MSC: Primary 47H05; secondary 47J20; 49J40; 90C33

Keywords: system of generalized mixed variational inequalities; fixed point problems; nearly uniformly Lipschitzian mapping; three-step resolvent iterative algorithm; convergence
\end{abstract}

\section{Introduction}

Variational inequality theory, which was initially introduced by Stampacchia [1] in 1964, is a branch of applicable mathematics with a wide range of applications in industry, physical, regional, social, pure, and applied sciences. This field is dynamic and is experiencing an explosive growth in both theory and applications; as a consequence, research techniques and problems are drawn from various fields. Variational inequalities have been generalized and extended in different directions using the novel and innovative techniques. An important and useful generalization is called the mixed variational inequality, or the variational inequality of the second kind, involving the nonlinear term. For applications, numerical methods, and other aspects of variational inequalities, see, for example, [1-22] and the references therein. In recent years, much attention has been given to develop efficient and implementable numerical methods including projection method and its variant forms, Wiener-Hopf (normal) equations, linear approximation, auxiliary principle, and descent framework for solving variational inequalities and related optimization problems. It is well known that the projection method and its variant forms and Wiener-Hopf equations technique cannot be used to suggest and analyze iterative methods for solving mixed variational inequalities due to the presence of the nonlinear term. These facts motivated us to use the technique of resolvent operators, the origin of which can be traced back to

(c) 2013 Petrot and Balooee; licensee Springer. This is an Open Access article distributed under the terms of the Creative Commons Attribution License (http://creativecommons.org/licenses/by/2.0), which permits unrestricted use, distribution, and reproduction in any medium, provided the original work is properly cited. 
Martinet [11] and Brezis [4]. In this technique, the given operator is decomposed into the sum of two (or more) maximal monotone operators, whose resolvents are easier to evaluate than the resolvent of the original operator. Such a method is known as the operator splitting method. This can lead to the development of very efficient methods, since one can treat each part of the original operator independently. The operator splitting methods and related techniques have been analyzed and studied by many authors including Peaceman and Rachford [15], Lions and Mercier [9], Glowinski and Tallec [7], and Tseng [18]. For an excellent account of the alternating direction implicit (splitting) methods, see [2]. A useful feature of the forward-backward splitting method for solving the mixed variational inequalities is that the resolvent step involves the subdifferential of the proper, convex and lower semicontinuous part only and the other part facilitates the problem decomposition.

Equally important is the area of mathematical sciences known as the resolvent equations, which was introduced by Noor [12]. Noor [12] established the equivalence between the mixed variational inequalities and the resolvent equations using essentially the resolvent operator technique. The resolvent equations are being used to develop powerful and efficient numerical methods for solving the mixed variational inequalities and related optimization problems. It is worth mentioning that if the nonlinear term involving the mixed variational inequalities is the indicator function of a closed convex set in a Hilbert space, then the resolvent operator is equal to the projection operator.

On the other hand, related to the variational inequalities, we have the problem of finding the fixed points of nonexpansive mappings, which is the subject of current interest in functional analysis. It is natural to consider a unified approach to these two different problems. Motivated and inspired by the research going in this direction, Noor and Huang [14] considered the problem of finding a common element of the set of solutions of variational inequalities and the set of fixed points of nonexpansive mappings. It is well known that every nonexpansive mapping is a Lipschitzian mapping. Lipschitzian mappings have been generalized by various authors. Sahu [23] introduced and investigated nearly uniformly Lipschitzian mappings as generalization of Lipschitzian mappings.

In the present paper, we introduce and consider a new system of generalized nonlinear mixed variational inequalities involving six different nonlinear operators (SGNMVID). We first verify the equivalence between the SGNMVID and the fixed point problems, and then by this equivalent formulation, we discuss the existence and uniqueness of the solution of the SGNMVID. Applying nearly uniformly Lipschitzian mappings $S_{i}(i=1,2,3)$ and the aforesaid equivalent alternative formulation, we suggest and analyze some new three-step resolvent iterative algorithms with mixed errors for finding the element of the set of fixed points of the nearly uniformly Lipschitzian mapping $\mathcal{Q}=\left(S_{1}, S_{2}, S_{3}\right)$, which is the unique solution of the SGNMVID. Also, the convergence analysis of the suggested iterative algorithms under suitable conditions is studied. In the final section, some comments on the results related to a class of strongly monotone mappings are discussed. The results presented in this paper extend and improve some known results in the literature.

\section{Preliminaries and basic results}

Throughout this article, we let $\mathcal{H}$ be a real Hilbert space which is equipped with an inner product $\langle\cdot, \cdot\rangle$ and the corresponding norm $\|\cdot\|$. Let $T_{i}: \mathcal{H} \times \mathcal{H} \times \mathcal{H} \rightarrow \mathcal{H}$ and $g_{i}: \mathcal{H} \rightarrow \mathcal{H}$ $(i=1,2,3)$ be six nonlinear single-valued operators such that for each $i=1,2,3, g_{i}$ is an 
onto operator, and let $\partial \varphi_{i}$ denote the subdifferential of the function $\varphi_{i}(i=1,2,3)$, where for each $i=1,2,3, \varphi_{i}: \mathcal{H} \rightarrow \mathbb{R} \cup\{+\infty\}$ is a proper convex lower semicontinuous function on $\mathcal{H}$. For any given constants $\rho, \eta, \gamma>0$, we consider the problem of finding $x^{*}, y^{*}, z^{*} \in \mathcal{H}$ such that

$$
\begin{cases}\left\langle\rho T_{1}\left(y^{*}, z^{*}, x^{*}\right)+x^{*}-g_{1}\left(y^{*}\right), g_{1}(x)-x^{*}\right\rangle \geq \rho \varphi_{1}\left(x^{*}\right)-\rho \varphi_{1}\left(g_{1}(x)\right), & \forall x \in \mathcal{H}, \\ \left\langle\eta T_{2}\left(z^{*}, x^{*}, y^{*}\right)+y^{*}-g_{2}\left(z^{*}\right), g_{2}(x)-y^{*}\right\rangle \geq \eta \varphi_{2}\left(y^{*}\right)-\eta \varphi_{2}\left(g_{2}(x)\right), & \forall x \in \mathcal{H}, \\ \left\langle\gamma T_{3}\left(x^{*}, y^{*}, z^{*}\right)+z^{*}-g_{3}\left(x^{*}\right), g_{3}(x)-z^{*}\right\rangle \geq \gamma \varphi_{3}\left(z^{*}\right)-\gamma \varphi_{3}\left(g_{3}(x)\right), & \forall x \in \mathcal{H},\end{cases}
$$

which is called the system of generalized nonlinear mixed variational inequalities involving six different nonlinear operators (SGNMVID).

If for each $i=1,2,3, g_{i} \equiv I$, the identity operator, and $\varphi_{i}(x)=\delta_{K}(x)$, for all $x \in K$, where $\delta_{K}$ is the indicator function of a nonempty closed convex set $K$ in $\mathcal{H}$ defined by

$$
\delta_{K}(y)= \begin{cases}0, & y \in K \\ \infty, & y \notin K\end{cases}
$$

then problem (2.1) reduces to the following system:

$$
\begin{cases}\left\langle\rho T_{1}\left(y^{*}, z^{*}, x^{*}\right)+x^{*}-y^{*}, x-x^{*}\right\rangle \geq 0, & \forall x \in K \\ \left\langle\eta T_{2}\left(z^{*}, x^{*}, y^{*}\right)+y^{*}-z^{*}, x-y^{*}\right\rangle \geq 0, & \forall x \in K \\ \left\langle\gamma T_{3}\left(x^{*}, y^{*}, z^{*}\right)+z^{*}-x^{*}, x-z^{*}\right\rangle \geq 0, & \forall x \in K\end{cases}
$$

which was introduced and studied by Cho and Qin [6].

For different choices of operators and constants, we obtain different systems and problems considered and studied in $[1,5,8,13,17,19-21]$ and the references therein.

Definition 2.1 A set-valued operator $T: \mathcal{H} \multimap \mathcal{H}$ is said to be monotone if, for any $x, y \in \mathcal{H}$,

$$
\langle u-v, x-y\rangle \geq 0, \quad \forall u \in T(x), v \in T(y) .
$$

A monotone set-valued operator $T$ is called maximal if its graph, $\operatorname{Gph}(T):=\{(x, y) \in$ $\mathcal{H} \times \mathcal{H}: y \in T(x)\}$, is not properly contained in the graph of any other monotone operator. It is well known that $T$ is a maximal monotone operator if and only if $(I+\lambda T)(\mathcal{H})=\mathcal{H}$ for all $\lambda>0$, where $I$ denotes the identity operator on $\mathcal{H}$.

Definition 2.2 [4] For any maximal monotone operator $T$, the resolvent operator associated with $T$ of parameter $\lambda$ is defined as

$$
J_{T}^{\lambda}(u)=(I+\lambda T)^{-1}(u), \quad \forall u \in \mathcal{H} .
$$

It is single-valued and nonexpansive, that is,

$$
\left\|J_{T}^{\lambda}(u)-J_{T}^{\lambda}(v)\right\| \leq\|u-v\|, \quad \forall u, v \in \mathcal{H} .
$$


If $\varphi$ is a proper, convex and lower-semicontinuous function, then its subdifferential $\partial \varphi$ is a maximal monotone operator, see Theorem 4 in [24]. In this case, we can define the resolvent operator associated with the subdifferential $\partial \varphi$ of parameter $\lambda$ as follows:

$$
J_{\varphi}^{\lambda}(u)=(I+\lambda \partial \varphi)^{-1}(u), \quad \forall u \in \mathcal{H}
$$

The resolvent operator $J_{\varphi}^{\lambda}$ has the following useful characterization.

Lemma 2.1 [13] For a given $z \in \mathcal{H}, x \in \mathcal{H}$ satisfies the inequality

$$
\langle x-z, y-x\rangle+\lambda \varphi(y)-\lambda \varphi(x) \geq 0, \quad \forall y \in \mathcal{H},
$$

if and only if $x=J_{\varphi}^{\lambda}(z)$, where $J_{\varphi}^{\lambda}$ is the resolvent operator associated with $\partial \varphi$ of parameter $\lambda>0$.

It is well known that $J_{\varphi}^{\lambda}$ is nonexpansive, that is,

$$
\left\|J_{\varphi}^{\lambda}(u)-J_{\varphi}^{\lambda}(v)\right\| \leq\|u-v\|, \quad \forall u, v \in \mathcal{H} .
$$

Let us recall that a mapping $T: \mathcal{H} \rightarrow \mathcal{H}$ is nonexpansive if $\|T x-T y\| \leq\|x-y\|$ for all $x, y \in \mathcal{H}$. In recent years, nonexpansive mappings have been generalized and investigated by various authors. In the next definitions, several generalizations of nonexpansive mappings are stated.

Definition 2.3 A nonlinear mapping $T: \mathcal{H} \rightarrow \mathcal{H}$ is called

(a) L-Lipschitzian if there exists a constant $L>0$ such that

$$
\|T x-T y\| \leq L\|x-y\|, \quad \forall x, y \in \mathcal{H} ;
$$

(b) generalized Lipschitzian [25] if there exists a constant $L>0$ such that

$$
\|T x-T y\| \leq L(\|x-y\|+1), \quad \forall x, y \in \mathcal{H} ;
$$

(c) generalized (L,M)-Lipschitzian [23] if there exist two constants $L, M>0$ such that

$$
\|T x-T y\| \leq L(\|x-y\|+M), \quad \forall x, y \in \mathcal{H} ;
$$

(d) asymptotically nonexpansive [26] if there exists a sequence $\left\{k_{n}\right\} \subseteq[1, \infty)$ with $\lim _{n \rightarrow \infty} k_{n}=1$ such that for each $n \in \mathbb{N}$,

$$
\left\|T^{n} x-T^{n} y\right\| \leq k_{n}\|x-y\|, \quad \forall x, y \in \mathcal{H} ;
$$

(e) pointwise asymptotically nonexpansive [27] if, for each integer $n \geq 1$,

$$
\left\|T^{n} x-T^{n} y\right\| \leq \alpha_{n}(x)\|x-y\|, \quad x, y \in \mathcal{H},
$$

where $\alpha_{n} \rightarrow 1$ pointwise on $X$; 
(f) uniformly L-Lipschitzian if there exists a constant $L>0$ such that for each $n \in \mathbb{N}$,

$$
\left\|T^{n} x-T^{n} y\right\| \leq L\|x-y\|, \quad \forall x, y \in \mathcal{H} .
$$

Definition 2.4 [23] A nonlinear mapping $T: \mathcal{H} \rightarrow \mathcal{H}$ is said to be

(a) nearly Lipschitzian with respect to the sequence $\left\{a_{n}\right\}$ if for each $n \in \mathbb{N}$, there exists a constant $k_{n}>0$ such that

$$
\left\|T^{n} x-T^{n} y\right\| \leq k_{n}\left(\|x-y\|+a_{n}\right), \quad \forall x, y \in \mathcal{H},
$$

where $\left\{a_{n}\right\}$ is a fix sequence in $[0, \infty)$ with $a_{n} \rightarrow 0$, as $n \rightarrow \infty$.

For an arbitrary, but fixed $n \in \mathbb{N}$, the infimum of constants $k_{n}$ in (2.3) is called nearly Lipschitz constant and is denoted by $\eta\left(T^{n}\right)$. Notice that

$$
\eta\left(T^{n}\right)=\sup \left\{\frac{\left\|T^{n} x-T^{n} y\right\|}{\|x-y\|+a_{n}}: x, y \in \mathcal{H}, x \neq y\right\}
$$

Definition 2.5 [23] A nearly Lipschitzian mapping $T$ with the sequence $\left\{\left(a_{n}, \eta\left(T^{n}\right)\right)\right\}$ is said to be

(a) nearly nonexpansive if $\eta\left(T^{n}\right)=1$ for all $n \in \mathbb{N}$, that is,

$$
\left\|T^{n} x-T^{n} y\right\| \leq\|x-y\|+a_{n}, \quad \forall x, y \in \mathcal{H}
$$

(b) nearly asymptotically nonexpansive if $\eta\left(T^{n}\right) \geq 1$ for all $n \in \mathbb{N}$ and $\lim _{n \rightarrow \infty} \eta\left(T^{n}\right)=1$, in other words, $k_{n} \geq 1$ for all $n \in \mathbb{N}$ with $\lim _{n \rightarrow \infty} k_{n}=1$;

(c) nearly uniformly L-Lipschitzian if $\eta\left(T^{n}\right) \leq L$ for all $n \in \mathbb{N}$, in other words, $k_{n}=L$ for all $n \in \mathbb{N}$.

Remark 2.2 It should be pointed out that:

(a) Every nonexpansive mapping is an asymptotically nonexpansive mapping, and every asymptotically nonexpansive mapping is a pointwise asymptotically nonexpansive mapping. Also, the class of Lipschitzian mappings properly includes the class of pointwise asymptotically nonexpansive mappings.

(b) It is obvious that every Lipschitzian mapping is a generalized Lipschitzian mapping. Furthermore, every mapping with a bounded range is a generalized Lipschitzian mapping. It is easy to see that the class of generalized $(L, M)$-Lipschitzian mappings is more general than the class of generalized Lipschitzian mappings.

(c) Clearly, the class of nearly uniformly $L$-Lipschitzian mappings properly includes the class of generalized $(L, M)$-Lipschitzian mappings and that of uniformly $L$-Lipschitzian mappings. Note that every nearly asymptotically nonexpansive mapping is nearly uniformly $L$-Lipschitzian.

Some interesting examples to investigate relations between the mappings given in Definitions 2.3, 2.4 and 2.5 can be found in [3]. 


\section{Existence of solution and uniqueness}

In this section, we prove the existence and uniqueness theorem for a solution of the system of generalized nonlinear mixed variational inequalities (2.1). For this end, we need the following lemma, in which, by using the resolvent operator technique and Lemma 2.1, the equivalence between the system of generalized nonlinear mixed variational inequalities (2.1) and fixed point problems is stated.

Lemma 3.1 Let $T_{i}, g_{i}, \varphi_{i}(i=1,2,3), \rho, \eta$ and $\gamma$ be the same as in SGNMVID (2.1). Then $\left(x^{*}, y^{*}, z^{*}\right) \in \mathcal{H} \times \mathcal{H} \times \mathcal{H}$ is a solution of SGNMVID (2.1) if and only if

$$
\left\{\begin{array}{l}
x^{*}=J_{\varphi_{1}}^{\rho}\left(g_{1}\left(y^{*}\right)-\rho T_{1}\left(y^{*}, z^{*}, x^{*}\right)\right), \\
y^{*}=J_{\varphi_{2}}^{\eta}\left(g_{2}\left(z^{*}\right)-\eta T_{2}\left(z^{*}, x^{*}, y^{*}\right)\right), \\
z^{*}=J_{\varphi_{3}}^{\gamma}\left(g_{3}\left(x^{*}\right)-\gamma T_{3}\left(x^{*}, y^{*}, z^{*}\right)\right),
\end{array}\right.
$$

where $J_{\varphi_{1}}^{\rho}$ is the resolvent operator associated with $\partial \varphi_{1}$ of parameter $\rho, J_{\varphi_{2}}^{\eta}$ is the resolvent operator associated with $\partial \varphi_{2}$ of parameter $\eta$ and $J_{\varphi_{3}}^{\gamma}$ is the resolvent operator associated with $\partial \varphi_{3}$ of parameter $\gamma$.

Proof $\left(x^{*}, y^{*}, z^{*}\right) \in \mathcal{H} \times \mathcal{H} \times \mathcal{H}$ is a solution of SGNMVID (2.1) if and only if

$$
\left\{\begin{array}{l}
\left\langle x^{*}-\left(g_{1}\left(y^{*}\right)-\rho T_{1}\left(y^{*}, z^{*}, x^{*}\right)\right), g_{1}(x)-x^{*}\right\rangle+\rho \varphi_{1}\left(g_{1}(x)\right)-\rho \varphi_{1}\left(x^{*}\right) \\
\geq 0, \quad \forall x \in \mathcal{H}, \\
\left\langle y^{*}-\left(g_{2}\left(z^{*}\right)-\eta T_{2}\left(z^{*}, x^{*}, y^{*}\right)\right), g_{2}(x)-y^{*}\right\rangle+\eta \varphi_{2}\left(g_{2}(x)\right)-\eta \varphi_{2}\left(y^{*}\right) \\
\geq 0, \quad \forall x \in \mathcal{H}, \\
\left\langle z^{*}-\left(g_{3}\left(x^{*}\right)-\gamma T_{3}\left(x^{*}, y^{*}, z^{*}\right)\right), g_{3}(x)-z^{*}\right\rangle+\gamma \varphi_{3}\left(g_{3}(x)\right)-\gamma \varphi_{3}\left(z^{*}\right) \\
\geq 0, \quad \forall x \in \mathcal{H} .
\end{array}\right.
$$

Since for each $i=1,2,3, g_{i}$ is an onto operator, Lemma 2.1 implies that $\left(x^{*}, y^{*}, z^{*}\right) \in \mathcal{H} \times$ $\mathcal{H} \times \mathcal{H}$ is a solution of (3.2) if and only if

$$
\left\{\begin{array}{l}
x^{*}=J_{\varphi_{1}}^{\rho}\left(g_{1}\left(y^{*}\right)-\rho T_{1}\left(y^{*}, z^{*}, x^{*}\right)\right), \\
y^{*}=J_{\varphi_{2}}^{\eta}\left(g_{2}\left(z^{*}\right)-\eta T_{2}\left(z^{*}, x^{*}, y^{*}\right)\right), \\
z^{*}=J_{\varphi_{3}}^{\gamma}\left(g_{3}\left(x^{*}\right)-\gamma T_{3}\left(x^{*}, y^{*}, z^{*}\right)\right) .
\end{array}\right.
$$

This completes the proof.

Definition 3.1 Let $T: \mathcal{H} \times \mathcal{H} \times \mathcal{H} \rightarrow \mathcal{H}$ and $g: \mathcal{H} \rightarrow \mathcal{H}$ be two single-valued operators. Then the operator

(a) $T$ is called monotone in the first variable if

$$
\langle T(x, \cdot, \cdot)-T(y, \cdot, \cdot), x-y\rangle \geq 0, \quad \forall x, y \in \mathcal{H}
$$

(b) $T$ is called $r$-strongly monotone in the first variable if there exists a constant $r>0$ such that

$$
\langle T(x, \cdot, \cdot)-T(y, \cdot, \cdot), x-y\rangle \geq r\|x-y\|^{2}, \quad \forall x, y \in \mathcal{H} ;
$$


(c) $T$ is called $(\kappa, \theta)$-relaxed cocoercive in the first variable if there exist two constants $\kappa, \theta>0$ such that

$$
\langle T(x, \cdot, \cdot)-T(y, \cdot, \cdot), x-y\rangle \geq-\kappa\|T(x, \cdot, \cdot)-T(y, \cdot, \cdot)\|^{2}+\theta\|x-y\|^{2}, \quad \forall x, y \in \mathcal{H}
$$

(d) $T$ is said to be $\mu$-Lipschitz continuous in the first variable if there exists a constant $\mu>0$ such that

$$
\|T(x, \cdot, \cdot)-T(y, \cdot, \cdot)\| \leq \mu\|x-y\|, \quad \forall x, y \in \mathcal{H}
$$

(e) $g$ is called $\gamma$-Lipschitz continuous if there exists a constant $\gamma>0$ such that

$$
\|g(x)-g(y)\| \leq \gamma\|x-y\|, \quad \forall x, y \in \mathcal{H}
$$

(f) $g$ is said to be $v$-strongly monotone if there exists a constant $v>0$ such that

$$
\langle g(x)-g(y), x-y\rangle \geq v\|x-y\|^{2}, \quad \forall x, y \in \mathcal{H} .
$$

Theorem 3.2 Let $T_{i}, g_{i}, \varphi_{i}(i=1,2,3), \rho, \eta$ and $\gamma$ be the same as in SGNMVID (2.1) such that for each $i=1,2,3, T_{i}$ is $\varsigma_{i}$-strongly monotone and $\sigma_{i}$-Lipschitz continuous in the first variable and $g_{i}$ is $\pi_{i}$-strongly monotone and $\delta_{i}$-Lipschitz continuous. If the constants $\rho, \eta$ and $\gamma$ satisfy the following conditions:

$$
\left\{\begin{array}{l}
\left|\rho-\frac{\varsigma_{1}}{\sigma_{1}^{2}}\right|<\frac{\sqrt{\varsigma_{1}^{2}-\sigma_{1}^{2} \mu_{1}\left(2-\mu_{1}\right)}}{\sigma_{1}^{2}}, \\
\left|\eta-\frac{\varsigma_{2}}{\sigma_{2}^{2}}\right|<\frac{\sqrt{\varsigma_{2}^{2}-\sigma_{2}^{2} \mu_{2}\left(2-\mu_{2}\right)}}{\sigma_{2}^{2}}, \\
\left|\gamma-\frac{\varsigma_{3}}{\sigma_{3}^{2}}\right|<\frac{\sqrt{\varsigma_{3}^{2}-\sigma_{3}^{2} \mu_{3}\left(2-\mu_{3}\right)}}{\sigma_{3}^{2}}, \\
\varsigma_{i}>\sigma_{i} \sqrt{\mu_{i}\left(2-\mu_{i}\right)} \quad(i=1,2,3), \\
\mu_{i}=\sqrt{1-\left(2 \pi_{i}-\delta_{i}^{2}\right)}<1 \quad(i=1,2,3), \\
2 \pi_{i}<1+\delta_{i}^{2} \quad(i=1,2,3),
\end{array}\right.
$$

then SGNMVID (2.1) admits a unique solution.

Proof Define the mappings $\Psi, \Phi, \Theta: \mathcal{H} \times \mathcal{H} \times \mathcal{H} \rightarrow \mathcal{H}$ by

$$
\begin{aligned}
& \Psi(x, y, z)=J_{\varphi_{1}}^{\rho}\left(g_{1}(y)-\rho T_{1}(y, z, x)\right), \\
& \Phi(x, y, z)=J_{\varphi_{2}}^{\eta}\left(g_{2}(z)-\eta T_{2}(z, x, y)\right), \\
& \Theta(x, y, z)=J_{\varphi_{3}}^{\gamma}\left(g_{3}(x)-\gamma T_{3}(x, y, z)\right),
\end{aligned}
$$

for all $(x, y, z) \in \mathcal{H} \times \mathcal{H} \times \mathcal{H}$. Define $\|\cdot\|_{*}$ on $\mathcal{H} \times \mathcal{H} \times \mathcal{H}$ by

$$
\|(x, y, z)\|_{*}=\|x\|+\|y\|+\|z\|, \quad \forall(x, y, z) \in \mathcal{H} \times \mathcal{H} \times \mathcal{H} .
$$

It is obvious that $\left(\mathcal{H} \times \mathcal{H} \times \mathcal{H},\|\cdot\|_{*}\right)$ is a Banach space. Moreover, define $F: \mathcal{H} \times \mathcal{H} \times \mathcal{H} \rightarrow$ $\mathcal{H} \times \mathcal{H} \times \mathcal{H}$ as follows:

$$
F(x, y, z)=(\Psi(x, y, z), \Phi(x, y, z), \Theta(x, y, z)), \quad \forall(x, y, z) \in \mathcal{H} \times \mathcal{H} \times \mathcal{H}
$$


Now, we prove that $F$ is a contraction mapping. For this end, let $(x, y, z),(\hat{x}, \hat{y}, \hat{z}) \in \mathcal{H} \times \mathcal{H} \times$ $\mathcal{H}$ be given. By using the nonexpansivity property of the resolvent operator $J_{\varphi_{1}}^{\rho}$, we get

$$
\begin{aligned}
& \|\Psi(x, y, z)-\Psi(\hat{x}, \hat{y}, \hat{z})\| \\
& \quad=\left\|J_{\varphi_{1}}^{\rho}\left(g_{1}(y)-\rho T_{1}(y, z, x)\right)-J_{\varphi_{1}}^{\rho}\left(g_{1}(\hat{y})-\rho T_{1}(\hat{y}, \hat{z}, \hat{x})\right)\right\| \\
& \quad \leq\left\|g_{1}(y)-g_{1}(\hat{y})-\rho\left(T_{1}(y, z, x)-T_{1}(\hat{y}, \hat{z}, \hat{x})\right)\right\| \\
& \quad \leq\left\|y-\hat{y}-\left(g_{1}(y)-g_{1}(\hat{y})\right)\right\|+\left\|y-\hat{y}-\rho\left(T_{1}(y, z, x)-T_{1}(\hat{y}, \hat{z}, \hat{x})\right)\right\| .
\end{aligned}
$$

Because $g_{1}$ is $\pi_{1}$-strongly monotone and $\delta_{1}$-Lipschitz continuous, we have

$$
\begin{aligned}
\| y & -\hat{y}-\left(g_{1}(y)-g_{1}(\hat{y})\right) \|^{2} \\
& =\|y-\hat{y}\|^{2}-2\left(g_{1}(y)-g_{1}(\hat{y}), y-\hat{y}\right)+\left\|g_{1}(y)-g_{1}(\hat{y})\right\|^{2} \\
& \leq\left(1-2 \pi_{1}\right)\|y-\hat{y}\|^{2}+\left\|g_{1}(y)-g_{1}(\hat{y})\right\|^{2} \\
& \leq\left(1-2 \pi_{1}+\delta_{1}^{2}\right)\|y-\hat{y}\|^{2} .
\end{aligned}
$$

Since $T_{1}$ is $\varsigma_{1}$-strongly monotone and $\sigma_{1}$-Lipschitz continuous in the first variable, we conclude that

$$
\begin{aligned}
\| y & -\hat{y}-\rho\left(T_{1}(y, z, x)-T_{1}(\hat{y}, \hat{z}, \hat{x})\right) \|^{2} \\
& =\|y-\hat{y}\|^{2}-2 \rho\left(T_{1}(y, z, x)-T_{1}(\hat{y}, \hat{z}, \hat{x}), y-\hat{y}\right\rangle+\rho^{2}\left\|T_{1}(y, z, x)-T_{1}(\hat{y}, \hat{z}, \hat{x})\right\|^{2} \\
& \leq\left(1-2 \rho \varsigma_{1}\right)\|y-\hat{y}\|^{2}+\rho^{2}\left\|T_{1}(y, z, x)-T_{1}(\hat{y}, \hat{z}, \hat{x})\right\|^{2} \\
& \leq\left(1-2 \rho \varsigma_{1}+\rho^{2} \sigma_{1}^{2}\right)\|y-\hat{y}\|^{2} .
\end{aligned}
$$

Substituting (3.7) and (3.8) in (3.6), we deduce that

$$
\|\Psi(x, y, z)-\Psi(\hat{x}, \hat{y}, \hat{z})\| \leq\left(\sqrt{1-2 \pi_{1}+\delta_{1}^{2}}+\sqrt{1-2 \rho \varsigma_{1}+\rho^{2} \sigma_{1}^{2}}\right)\|y-\hat{y}\| .
$$

Like in the proof of (3.9), we can establish that

$$
\|\Phi(x, y, z)-\Phi(\hat{x}, \hat{y}, \hat{z})\| \leq\left(\sqrt{1-2 \pi_{2}+\delta_{2}^{2}}+\sqrt{1-2 \eta \zeta_{2}+\eta^{2} \sigma_{2}^{2}}\right)\|z-\hat{z}\|
$$

and

$$
\|\Theta(x, y, z)-\Theta(\hat{x}, \hat{y}, \hat{z})\| \leq\left(\sqrt{1-2 \pi_{3}+\delta_{3}^{2}}+\sqrt{1-2 \gamma \varsigma_{3}+\gamma^{2} \sigma_{3}^{2}}\right)\|x-\hat{x}\| .
$$

From (3.9)-(3.11), it follows that

$$
\begin{aligned}
& \|\Psi(x, y, z)-\Psi(\hat{x}, \hat{y}, \hat{z})\|+\|\Phi(x, y, z)-\Phi(\hat{x}, \hat{y}, \hat{z})\|+\|\Theta(x, y, z)-\Theta(\hat{x}, \hat{y}, \hat{z})\| \\
& \quad \leq \vartheta\|x-\hat{x}\|+\theta\|y-\hat{y}\|+\varrho\|z-\hat{z}\|,
\end{aligned}
$$


where

$$
\begin{aligned}
& \vartheta=\sqrt{1-2 \pi_{3}+\delta_{3}^{2}}+\sqrt{1-2 \gamma \varsigma_{3}+\gamma^{2} \sigma_{3}^{2}}, \\
& \theta=\sqrt{1-2 \pi_{1}+\delta_{1}^{2}}+\sqrt{1-2 \rho \varsigma_{1}+\rho^{2} \sigma_{1}^{2}}, \\
& \varrho=\sqrt{1-2 \pi_{2}+\delta_{2}^{2}}+\sqrt{1-2 \eta \varsigma_{2}+\eta^{2} \sigma_{2}^{2}} .
\end{aligned}
$$

Applying (3.5) and (3.12), we conclude that

$$
\|F(x, y, z)-F(\hat{x}, \hat{y}, \hat{z})\|_{*} \leq \lambda\|(x, y, z)-(\hat{x}, \hat{y}, \hat{z})\|_{*^{\prime}}
$$

where $\lambda=\max \{\vartheta, \theta, \varrho\}$. Condition (3.3) implies that $0 \leq \lambda<1$ and so (3.14) guarantees that the mapping $F$ is contraction. According to the Banach fixed point theorem, there exists a unique point $\left(x^{*}, y^{*}, z^{*}\right) \in \mathcal{H} \times \mathcal{H} \times \mathcal{H}$ such that $F\left(x^{*}, y^{*}, z^{*}\right)=\left(x^{*}, y^{*}, z^{*}\right)$. It follows from (3.4) and (3.5) that $x^{*}=J_{\varphi_{1}}^{\rho}\left(g_{1}\left(y^{*}\right)-\rho T_{1}\left(y^{*}, z^{*}, x^{*}\right)\right), y^{*}=J_{\varphi_{2}}^{\eta}\left(g_{2}\left(z^{*}\right)-\eta T_{2}\left(z^{*}, x^{*}, y^{*}\right)\right)$ and $z^{*}=$ $J_{\varphi_{3}}^{\gamma}\left(g_{3}\left(x^{*}\right)-\gamma T_{3}\left(x^{*}, y^{*}, z^{*}\right)\right)$. Now, it follows from Lemma 3.1 that $\left(x^{*}, y^{*}, z^{*}\right) \in \mathcal{H} \times \mathcal{H} \times \mathcal{H}$ is a unique solution of SGNMVID (2.1). This completes the proof.

\section{Some new three-step resolvent iterative algorithms}

In this section, applying nearly uniformly Lipschitzian mappings $S_{i}(i=1,2,3)$ and by using the equivalent alternative formulation (3.1), we suggest and analyze some new three-step resolvent iterative algorithms with mixed errors for finding an element of the set of fixed points of $\mathcal{Q}=\left(S_{1}, S_{2}, S_{3}\right)$, which is the unique solution of SGNMVID (2.1).

Let $S_{1}: \mathcal{H} \rightarrow \mathcal{H}$ be a nearly uniformly $L_{1}$-Lipschitzian mapping with the sequence $\left\{a_{n}\right\}_{n=1}^{\infty}$, let $S_{2}: \mathcal{H} \rightarrow \mathcal{H}$ be a nearly uniformly $L_{2}$-Lipschitzian mapping with the sequence $\left\{b_{n}\right\}_{n=1}^{\infty}$ and let $S_{3}: \mathcal{H} \rightarrow \mathcal{H}$ be a nearly uniformly $L_{3}$-Lipschitzian mapping with the sequence $\left\{c_{n}\right\}_{n=1}^{\infty}$. We define the self-mapping $\mathcal{Q}$ of $\mathcal{H} \times \mathcal{H} \times \mathcal{H}$ as follows:

$$
\mathcal{Q}(x, y, z)=\left(S_{1} x, S_{2} y, S_{3} z\right), \quad \forall x, y, z \in \mathcal{H} .
$$

Then $\mathcal{Q}=\left(S_{1}, S_{2}, S_{3}\right): \mathcal{H} \times \mathcal{H} \times \mathcal{H} \rightarrow \mathcal{H} \times \mathcal{H} \times \mathcal{H}$ is a nearly uniformly $\max \left\{L_{1}, L_{2}, L_{3}\right\}-$ Lipschitzian mapping with the sequence $\left\{a_{n}+b_{n}+c_{n}\right\}_{n=1}^{\infty}$ with respect to the norm $\|\cdot\|_{*}$ in $\mathcal{H} \times \mathcal{H} \times \mathcal{H}$. To see this fact, let $(x, y, z),\left(x^{\prime}, y^{\prime}, z^{\prime}\right) \in \mathcal{H} \times \mathcal{H} \times \mathcal{H}$ be arbitrary. Then, for any $n \in \mathbb{N}$, we have

$$
\begin{aligned}
& \left\|\mathcal{Q}^{n}(x, y, z)-\mathcal{Q}^{n}\left(x^{\prime}, y^{\prime}, z^{\prime}\right)\right\|_{*} \\
& \quad=\left\|\left(S_{1}^{n} x, S_{2}^{n} y, S_{3}^{n} z\right)-\left(S_{1}^{n} x^{\prime}, S_{2}^{n} y^{\prime}, S_{3}^{n} z^{\prime}\right)\right\|_{*} \\
& \quad=\left\|\left(S_{1}^{n} x-S_{1}^{n} x^{\prime}, S_{2}^{n} y-S_{2}^{n} y^{\prime}, S_{3}^{n} z-S_{3}^{n} z^{\prime}\right)\right\|_{*} \\
& \quad=\left\|S_{1}^{n} x-S_{1}^{n} x^{\prime}\right\|+\left\|S_{2}^{n} y-S_{2}^{n} y^{\prime}\right\|+\left\|S_{3}^{n} z-S_{3}^{n} z^{\prime}\right\| \\
& \quad \leq L_{1}\left(\left\|x-x^{\prime}\right\|+a_{n}\right)+L_{2}\left(\left\|y-y^{\prime}\right\|+b_{n}\right)+L_{3}\left(\left\|z-z^{\prime}\right\|+c_{n}\right) \\
& \quad \leq \max \left\{L_{1}, L_{2}, L_{3}\right\}\left(\left\|x-x^{\prime}\right\|+\left\|y-y^{\prime}\right\|+\left\|z-z^{\prime}\right\|+a_{n}+b_{n}+c_{n}\right) \\
& \quad=\max \left\{L_{1}, L_{2}, L_{3}\right\}\left(\left\|(x, y, z)-\left(x^{\prime}, y^{\prime}, z^{\prime}\right)\right\|_{*}+a_{n}+b_{n}+c_{n}\right) .
\end{aligned}
$$


We denote the sets of all the fixed points of $S_{i}(i=1,2,3)$ and $\mathcal{Q}$ by $\operatorname{Fix}\left(S_{i}\right)$ and $\operatorname{Fix}(\mathcal{Q})$, respectively, and the set of all the solutions of system (2.1) by $\operatorname{SGNMVID}\left(\mathcal{H}, T_{i}, g_{i}, \varphi_{i}, i=\right.$ $1,2,3)$. It is clear that for any $(x, y, z) \in \mathcal{H} \times \mathcal{H} \times \mathcal{H},(x, y, z) \in \operatorname{Fix}(\mathcal{Q})$ if and only if $x \in$ $\operatorname{Fix}\left(S_{1}\right), y \in \operatorname{Fix}\left(S_{2}\right)$ and $z \in \operatorname{Fix}\left(S_{3}\right)$, that is, $\operatorname{Fix}(\mathcal{Q})=\operatorname{Fix}\left(S_{1}, S_{2}, S_{3}\right)=\operatorname{Fix}\left(S_{1}\right) \times \operatorname{Fix}\left(S_{2}\right) \times$ $\operatorname{Fix}\left(S_{3}\right)$. We now characterize the problem. Let $T_{i}, g_{i}, \varphi_{i}(i=1,2,3), \rho, \eta$ and $\gamma$ be the same as in $\operatorname{SGNMVID~(2.1).~If~}\left(x^{*}, y^{*}, z^{*}\right) \in \operatorname{Fix}(\mathcal{Q}) \cap \operatorname{SGNMVID}\left(\mathcal{H}, T_{i}, g_{i}, \varphi_{i}, i=1,2,3\right)$, then $x^{*} \in \operatorname{Fix}\left(S_{1}\right), y^{*} \in \operatorname{Fix}\left(S_{2}\right), z^{*} \in \operatorname{Fix}\left(S_{3}\right)$ and $\left(x^{*}, y^{*}, z^{*}\right) \in \operatorname{SGNMVID}\left(\mathcal{H}, T_{i}, g_{i}, \varphi_{i}, i=1,2,3\right)$. Therefore, it follows from Lemma 3.1 that for each $n \in \mathbb{N}$,

$$
\left\{\begin{array}{l}
x^{*}=S_{1}^{n} x^{*}=J_{\varphi_{1}}^{\rho}\left(g_{1}\left(y^{*}\right)-\rho T_{1}\left(y^{*}, z^{*}, x^{*}\right)\right)=S_{1}^{n} J_{\varphi_{1}}^{\rho}\left(g_{1}\left(y^{*}\right)-\rho T_{1}\left(y^{*}, z^{*}, x^{*}\right)\right), \\
y^{*}=S_{2}^{n} y^{*}=J_{\varphi_{2}}^{\eta}\left(g_{2}\left(z^{*}\right)-\eta T_{2}\left(z^{*}, x^{*}, y^{*}\right)\right)=S_{2}^{n} J_{\varphi_{2}}^{\eta}\left(g_{2}\left(z^{*}\right)-\eta T_{2}\left(z^{*}, x^{*}, y^{*}\right)\right), \\
z^{*}=S_{3}^{n} z^{*}=J_{\varphi_{3}}^{\gamma}\left(g_{3}\left(x^{*}\right)-\gamma T_{3}\left(x^{*}, y^{*}, z^{*}\right)\right)=S_{3}^{n} J_{\varphi_{3}}^{\gamma}\left(g_{3}\left(x^{*}\right)-\gamma T_{3}\left(x^{*}, y^{*}, z^{*}\right)\right) .
\end{array}\right.
$$

The fixed point formulation (4.2) is used to suggest the following three-step resolvent iterative algorithm with mixed errors for finding an element of the set of fixed points of the nearly uniformly Lipschitzian mapping $\mathcal{Q}=\left(S_{1}, S_{2}, S_{3}\right)$, which is a unique solution of SGNMVID (2.1).

Algorithm 4.1 Let $T_{i}, g_{i}, \varphi_{i}(i=1,2,3), \rho, \eta$ and $\gamma$ be the same as in SGNMVID (2.1). For an arbitrary chosen initial point $\left(x_{1}, y_{1}, z_{1}\right) \in \mathcal{H} \times \mathcal{H} \times \mathcal{H}$, compute the iterative sequence $\left\{\left(x_{n}, y_{n}, z_{n}\right)\right\}_{n=1}^{\infty}$ by the iterative processes

$$
\left\{\begin{aligned}
x_{n+1}= & \left(1-\alpha_{n}-\beta_{n}\right) x_{n}+\alpha_{n} S_{1}^{n} J_{\varphi_{1}}^{\rho}\left(g_{1}\left(y_{n+1}\right)-\rho T_{1}\left(y_{n+1}, z_{n+1}, x_{n}\right)\right) \\
& +\alpha_{n} e_{n}+\beta_{n} j_{n}+r_{n} \\
y_{n+1}= & \left(1-\alpha_{n}^{\prime}-\beta_{n}^{\prime}\right) x_{n}+\alpha_{n}^{\prime} S_{2}^{n} J_{\varphi_{2}}^{\eta}\left(g_{2}\left(z_{n+1}\right)-\eta T_{2}\left(z_{n+1}, x_{n}, y_{n}\right)\right) \\
& +\alpha_{n}^{\prime} p_{n}+\beta_{n}^{\prime} q_{n}+k_{n} \\
z_{n+1}= & \left(1-\alpha_{n}^{\prime \prime}-\beta_{n}^{\prime \prime}\right) x_{n}+\alpha_{n}^{\prime \prime} S_{3}^{n} J_{\varphi_{3}}^{\gamma}\left(g_{3}\left(x_{n}\right)-\gamma T_{3}\left(x_{n}, y_{n}, z_{n}\right)\right) \\
& +\alpha_{n}^{\prime \prime} s_{n}+\beta_{n}^{\prime \prime} t_{n}+l_{n}
\end{aligned}\right.
$$

where $S_{i}: \mathcal{H} \rightarrow \mathcal{H}(i=1,2,3)$ are three nearly uniformly Lipschitzian mappings, $\left\{\alpha_{n}\right\}_{n=1}^{\infty}$, $\left\{\alpha_{n}^{\prime}\right\}_{n=1}^{\infty},\left\{\alpha_{n}^{\prime \prime}\right\}_{n=1}^{\infty},\left\{\beta_{n}\right\}_{n=1}^{\infty},\left\{\beta_{n}^{\prime}\right\}_{n=1}^{\infty}$ and $\left\{\beta_{n}^{\prime \prime}\right\}_{n=1}^{\infty}$ are sequences in the interval $[0,1]$ such that $\sum_{n=1}^{\infty} \alpha_{n}=\infty, \sum_{n=1}^{\infty} \beta_{n}<\infty, \sum_{n=1}^{\infty} \beta_{n}^{\prime}<\infty, \sum_{n=1}^{\infty} \beta_{n}^{\prime \prime}<\infty, \alpha_{n}+\beta_{n} \leq 1, \alpha_{n}^{\prime}+\beta_{n}^{\prime} \leq 1, \alpha_{n}^{\prime \prime}+\beta_{n}^{\prime \prime} \leq$ $1, \lim _{n \rightarrow \infty} \alpha_{n}^{\prime}=1, \lim _{n \rightarrow \infty} \alpha_{n}^{\prime \prime}=1$ and $\left\{e_{n}\right\}_{n=1}^{\infty},\left\{p_{n}\right\}_{n=1}^{\infty},\left\{s_{n}\right\}_{n=1}^{\infty},\left\{j_{n}\right\}_{n=1}^{\infty},\left\{q_{n}\right\}_{n=1}^{\infty},\left\{t_{n}\right\}_{n=1}^{\infty},\left\{r_{n}\right\}_{n=1}^{\infty}$, $\left\{k_{n}\right\}_{n=1}^{\infty},\left\{l_{n}\right\}_{n=1}^{\infty}$ are nine sequences in $\mathcal{H}$ to take into account a possible inexact computation of the resolvent operator point satisfying the following conditions:

$$
\left\{\begin{array}{l}
e_{n}=e_{n}^{\prime}+e_{n}^{\prime \prime}, \quad p_{n}=p_{n}^{\prime}+p_{n}^{\prime \prime}, \quad s_{n}=s_{n}^{\prime}+s_{n}^{\prime \prime}, \\
\lim _{n \rightarrow \infty}\left\|e_{n}^{\prime}\right\|=0, \quad \lim _{n \rightarrow \infty}\left\|p_{n}^{\prime}\right\|=0, \quad \lim _{n \rightarrow \infty}\left\|s_{n}^{\prime}\right\|=0, \\
\sum_{n=1}^{\infty}\left\|e_{n}^{\prime \prime}\right\|<\infty, \quad \sum_{n=1}^{\infty}\left\|p_{n}^{\prime \prime}\right\|<\infty, \quad \sum_{n=1}^{\infty}\left\|s_{n}^{\prime \prime}\right\|<\infty, \\
\sum_{n=1}^{\infty}\left\|r_{n}\right\|<\infty, \quad \sum_{n=1}^{\infty}\left\|k_{n}\right\|<\infty, \quad \sum_{n=1}^{\infty}\left\|l_{n}\right\|<\infty
\end{array}\right.
$$

If for each $i=1,2,3, S_{i} \equiv I$, then Algorithm 4.1 reduces to the following algorithm.

Algorithm 4.2 Let $T_{i}, g_{i}, \varphi_{i}(i=1,2,3), \rho, \eta$ and $\gamma$ be the same as in SGNMVID (2.1). For an arbitrary chosen initial point $\left(x_{1}, y_{1}, z_{1}\right) \in \mathcal{H} \times \mathcal{H} \times \mathcal{H}$, compute the iterative sequence 
$\left\{\left(x_{n}, y_{n}, z_{n}\right)\right\}_{n=1}^{\infty}$ in the following way:

$$
\left\{\begin{array}{l}
x_{n+1}=\left(1-\alpha_{n}-\beta_{n}\right) x_{n}+\alpha_{n} J_{\varphi_{1}}^{\rho}\left(g_{1}\left(y_{n+1}\right)-\rho T_{1}\left(y_{n+1}, z_{n+1}, x_{n}\right)\right)+\alpha_{n} e_{n}+\beta_{n} j_{n}+r_{n}, \\
y_{n+1}=\left(1-\alpha_{n}^{\prime}-\beta_{n}^{\prime}\right) x_{n}+\alpha_{n}^{\prime} J_{\varphi_{2}}^{\eta}\left(g_{2}\left(z_{n+1}\right)-\eta T_{2}\left(z_{n+1}, x_{n}, y_{n}\right)\right)+\alpha_{n}^{\prime} p_{n}+\beta_{n}^{\prime} q_{n}+k_{n}, \\
z_{n+1}=\left(1-\alpha_{n}^{\prime \prime}-\beta_{n}^{\prime \prime}\right) x_{n}+\alpha_{n}^{\prime \prime} J_{\varphi_{3}}^{\gamma}\left(g_{3}\left(x_{n}\right)-\gamma T_{3}\left(x_{n}, y_{n}, z_{n}\right)\right)+\alpha_{n}^{\prime \prime} s_{n}+\beta_{n}^{\prime \prime} t_{n}+l_{n},
\end{array}\right.
$$

where the sequences $\left\{\alpha_{n}\right\}_{n=1}^{\infty},\left\{\alpha_{n}^{\prime}\right\}_{n=1}^{\infty},\left\{\alpha_{n}^{\prime \prime}\right\}_{n=1}^{\infty},\left\{\beta_{n}\right\}_{n=1}^{\infty},\left\{\beta_{n}^{\prime}\right\}_{n=1}^{\infty},\left\{\beta_{n}^{\prime \prime}\right\}_{n=1}^{\infty},\left\{e_{n}\right\}_{n=1}^{\infty},\left\{p_{n}\right\}_{n=1}^{\infty}$, $\left\{s_{n}\right\}_{n=1}^{\infty},\left\{j_{n}\right\}_{n=1}^{\infty},\left\{q_{n}\right\}_{n=1}^{\infty},\left\{t_{n}\right\}_{n=1}^{\infty},\left\{r_{n}\right\}_{n=1}^{\infty},\left\{k_{n}\right\}_{n=1}^{\infty}$ and $\left\{l_{n}\right\}_{n=1}^{\infty}$ are the same as in Algorithm 4.1.

Remark 4.3 Equality (4.2) can be written as follows:

$$
\left\{\begin{array}{l}
x^{*}=S_{1}^{n} J_{\varphi_{1}}^{\rho}(u), \quad y^{*}=S_{2}^{n} J_{\varphi_{2}}^{\eta}(v), \quad z^{*}=S_{3}^{n} J_{\varphi_{3}}^{\gamma}(w), \\
u=g_{1}\left(y^{*}\right)-\rho T_{1}\left(y^{*}, z^{*}, x^{*}\right), \quad v=g_{2}\left(z^{*}\right)-\eta T_{2}\left(z^{*}, x^{*}, y^{*}\right), \\
w=g_{3}\left(x^{*}\right)-\gamma T_{3}\left(x^{*}, y^{*}, z^{*}\right) .
\end{array}\right.
$$

The fixed point formulation (4.5) enables us to suggest the following iterative algorithms.

Algorithm 4.4 Let $T_{i}, g_{i}, \varphi_{i}(i=1,2,3), \rho, \eta$ and $\gamma$ be the same as in SGNMVID (2.1). For an arbitrary chosen initial point $\left(u_{1}, v_{1}, w_{1}\right) \in \mathcal{H} \times \mathcal{H} \times \mathcal{H}$, compute the iterative sequence $\left\{\left(x_{n}, y_{n}, z_{n}\right)\right\}_{n=1}^{\infty}$ in the following way:

$$
\left\{\begin{array}{l}
x_{n}=S_{1}^{n} J_{\varphi_{1}}^{\rho}\left(u_{n}\right), \quad y_{n}=S_{2}^{n} J_{\varphi_{2}}^{\eta}\left(v_{n}\right), \quad z_{n}=S_{3}^{n} J_{\varphi_{3}}^{\gamma}\left(w_{n}\right), \\
u_{n+1}=\left(1-\alpha_{n}-\beta_{n}\right) u_{n}+\alpha_{n}\left(g_{1}\left(y_{n}\right)-\rho T_{1}\left(y_{n}, z_{n}, x_{n}\right)\right)+\alpha_{n} e_{n}+\beta_{n} j_{n}+r_{n}, \\
v_{n+1}=\left(1-\alpha_{n}-\beta_{n}\right) v_{n}+\alpha_{n}\left(g_{2}\left(z_{n}\right)-\eta T_{2}\left(z_{n}, x_{n}, y_{n}\right)\right)+\alpha_{n} p_{n}+\beta_{n} q_{n}+k_{n}, \\
w_{n+1}=\left(1-\alpha_{n}-\beta_{n}\right) w_{n}+\alpha_{n}\left(g_{3}\left(x_{n}\right)-\gamma T_{3}\left(x_{n}, y_{n}, z_{n}\right)\right)+\alpha_{n} s_{n}+\beta_{n} t_{n}+l_{n},
\end{array}\right.
$$

where $S_{i}: \mathcal{H} \rightarrow \mathcal{H}(i=1,2,3)$ are three nearly uniformly Lipschitzian mappings, $\left\{\alpha_{n}\right\}_{n=1}^{\infty}$, $\left\{\beta_{n}\right\}_{n=1}^{\infty}$ are sequences in $[0,1]$ such that $\sum_{n=1}^{\infty} \alpha_{n}=\infty, \sum_{n=1}^{\infty} \beta_{n}<\infty, \alpha_{n}+\beta_{n} \leq 1$ and the sequences $\left\{e_{n}\right\}_{n=1}^{\infty},\left\{p_{n}\right\}_{n=1}^{\infty},\left\{s_{n}\right\}_{n=1}^{\infty},\left\{j_{n}\right\}_{n=1}^{\infty},\left\{q_{n}\right\}_{n=1}^{\infty},\left\{t_{n}\right\}_{n=1}^{\infty},\left\{r_{n}\right\}_{n=1}^{\infty},\left\{k_{n}\right\}_{n=1}^{\infty},\left\{l_{n}\right\}_{n=1}^{\infty}$ are the same as in Algorithm 4.1 satisfying (4.4).

If $\beta_{n}=0$, for all $n \in \mathbb{N}$, then Algorithm 4.4 reduces to the following algorithm.

Algorithm 4.5 Let $T_{i}, g_{i}, \varphi_{i}(i=1,2,3), \rho, \eta$ and $\gamma$ be the same as in SGNMVID (2.1). For an arbitrary chosen initial point $\left(u_{1}, v_{1}, w_{1}\right) \in \mathcal{H} \times \mathcal{H} \times \mathcal{H}$, compute the iterative sequence $\left\{\left(x_{n}, y_{n}, z_{n}\right)\right\}_{n=1}^{\infty}$ in the following way:

$$
\left\{\begin{array}{l}
x_{n}=S_{1}^{n} J_{\varphi_{1}}^{\rho}\left(u_{n}\right), \quad y_{n}=S_{2}^{n} J_{\varphi_{2}}^{\eta}\left(v_{n}\right), \quad z_{n}=S_{3}^{n} J_{\varphi_{3}}^{\gamma}\left(w_{n}\right), \\
u_{n+1}=\left(1-\alpha_{n}\right) u_{n}+\alpha_{n}\left(g_{1}\left(y_{n}\right)-\rho T_{1}\left(y_{n}, z_{n}, x_{n}\right)\right)+\alpha_{n} e_{n}+r_{n}, \\
v_{n+1}=\left(1-\alpha_{n}\right) v_{n}+\alpha_{n}\left(g_{2}\left(z_{n}\right)-\eta T_{2}\left(z_{n}, x_{n}, y_{n}\right)\right)+\alpha_{n} p_{n}+k_{n}, \\
w_{n+1}=\left(1-\alpha_{n}\right) w_{n}+\alpha_{n}\left(g_{3}\left(x_{n}\right)-\gamma T_{3}\left(x_{n}, y_{n}, z_{n}\right)\right)+\alpha_{n} s_{n}+l_{n},
\end{array}\right.
$$

where $S_{i}(i=1,2,3),\left\{\alpha_{n}\right\}_{n=1}^{\infty},\left\{e_{n}\right\}_{n=1}^{\infty},\left\{p_{n}\right\}_{n=1}^{\infty},\left\{s_{n}\right\}_{n=1}^{\infty},\left\{r_{n}\right\}_{n=1}^{\infty},\left\{k_{n}\right\}_{n=1}^{\infty}$ and $\left\{l_{n}\right\}_{n=1}^{\infty}$ are the same as in Algorithm 4.1. 
If $S_{i} \equiv I(i=1,2,3)$, then Algorithm 4.4 collapses to the following algorithm.

Algorithm 4.6 Let $T_{i}, g_{i}, \varphi_{i}(i=1,2,3), \rho, \eta$ and $\gamma$ be the same as in SGNMVID (2.1). For an arbitrary chosen initial point $\left(u_{1}, v_{1}, w_{1}\right) \in \mathcal{H} \times \mathcal{H} \times \mathcal{H}$, compute the iterative sequence $\left\{\left(x_{n}, y_{n}, z_{n}\right)\right\}_{n=1}^{\infty}$ in the following way:

$$
\left\{\begin{array}{l}
x_{n}=J_{\varphi_{1}}^{\rho}\left(u_{n}\right), \quad y_{n}=J_{\varphi_{2}}^{\eta}\left(v_{n}\right), \quad z_{n}=J_{\varphi_{3}}^{\gamma}\left(w_{n}\right), \\
u_{n+1}=\left(1-\alpha_{n}-\beta_{n}\right) u_{n}+\alpha_{n}\left(g_{1}\left(y_{n}\right)-\rho T_{1}\left(y_{n}, z_{n}, x_{n}\right)\right)+\alpha_{n} e_{n}+\beta_{n} j_{n}+r_{n}, \\
v_{n+1}=\left(1-\alpha_{n}-\beta_{n}\right) v_{n}+\alpha_{n}\left(g_{2}\left(z_{n}\right)-\eta T_{2}\left(z_{n}, x_{n}, y_{n}\right)\right)+\alpha_{n} p_{n}+\beta_{n} q_{n}+k_{n}, \\
w_{n+1}=\left(1-\alpha_{n}-\beta_{n}\right) w_{n}+\alpha_{n}\left(g_{3}\left(x_{n}\right)-\gamma T_{3}\left(x_{n}, y_{n}, z_{n}\right)\right)+\alpha_{n} s_{n}+\beta_{n} t_{n}+l_{n},
\end{array}\right.
$$

where the sequences $\left\{\alpha_{n}\right\}_{n=1}^{\infty},\left\{\beta_{n}\right\}_{n=1}^{\infty},\left\{e_{n}\right\}_{n=1}^{\infty},\left\{p_{n}\right\}_{n=1}^{\infty},\left\{s_{n}\right\}_{n=1}^{\infty},\left\{j_{n}\right\}_{n=1}^{\infty},\left\{q_{n}\right\}_{n=1}^{\infty},\left\{t_{n}\right\}_{n=1}^{\infty}$, $\left\{r_{n}\right\}_{n=1}^{\infty},\left\{k_{n}\right\}_{n=1}^{\infty}$ and $\left\{l_{n}\right\}_{n=1}^{\infty}$ are the same as in Algorithm 4.1.

\section{Main results}

In this section, we discuss the convergence analysis of the suggested three-step resolvent iterative algorithms under suitable conditions. For this end, we need the following lemma.

Lemma 5.1 Let $\left\{a_{n}\right\},\left\{b_{n}\right\}$ and $\left\{c_{n}\right\}$ be three nonnegative real sequences satisfying the following condition: There exists a natural number $n_{0}$ such that

$$
a_{n+1} \leq\left(1-t_{n}\right) a_{n}+b_{n} t_{n}+c_{n}, \quad \forall n \geq n_{0},
$$

where $t_{n} \in[0,1], \sum_{n=0}^{\infty} t_{n}=\infty, \lim _{n \rightarrow \infty} b_{n}=0, \sum_{n=0}^{\infty} c_{n}<\infty$. Then $\lim _{n \rightarrow 0} a_{n}=0$.

Proof The proof directly follows from Lemma 2 in Liu [10].

Theorem 5.2 Let $T_{i}, g_{i}, \varphi_{i}(i=1,2,3), \rho, \eta$ and $\gamma$ be the same as in Theorem 3.2 and let all the conditions of Theorem 3.2 hold. Suppose that $S_{1}: \mathcal{H} \rightarrow \mathcal{H}$ is a nearly uniformly $L_{1}$-Lipschitzian mapping with the sequence $\left\{b_{n}\right\}_{n=1}^{\infty}$, that $S_{2}: \mathcal{H} \rightarrow \mathcal{H}$ is a nearly uniformly $L_{2}$-Lipschitzian mapping with the sequence $\left\{c_{n}\right\}_{n=1}^{\infty}$, that $S_{3}: \mathcal{H} \rightarrow \mathcal{H}$ is a nearly uniformly $L_{3}$-Lipschitzian mapping with the sequence $\left\{d_{n}\right\}_{n=1}^{\infty}$, and that the selfmapping $\mathcal{Q}$ of $\mathcal{H} \times \mathcal{H} \times \mathcal{H}$ is defined by (4.1) such that $\operatorname{Fix}(\mathcal{Q}) \cap \operatorname{SGNMVID}\left(\mathcal{H}, T_{i}, g_{i}, \varphi_{i}, i=\right.$ $1,2,3) \neq \emptyset$. Further, let $L_{i} \lambda<1$, where $\lambda$ is the same as in (3.14). Then the iterative sequence $\left\{\left(x_{n}, y_{n}, z_{n}\right)\right\}_{n=1}^{\infty}$ generated by Algorithm 4.1, converges strongly to the only element of $\operatorname{Fix}(\mathcal{Q}) \cap \operatorname{SGNMVID}\left(\mathcal{H}, T_{i}, g_{i}, \varphi_{i}, i=1,2,3\right)$.

Proof According to Theorem 3.2, SGNMVID (2.1) has a unique solution $\left(x^{*}, y^{*}, z^{*}\right) \in \mathcal{H} \times$ $\mathcal{H} \times \mathcal{H}$. Accordingly, in view of Lemma 3.1, $\left(x^{*}, y^{*}, z^{*}\right)$ satisfies (3.1). Since $\operatorname{SGNMVID}\left(\mathcal{H}, T_{i}\right.$, $\left.g_{i}, \varphi_{i}, i=1,2,3\right)$ is a singleton set, it follows from $\operatorname{Fix}(\mathcal{Q}) \cap \operatorname{SGNMVID}\left(\mathcal{H}, T_{i}, g_{i}, \varphi_{i}, i=\right.$ $1,2,3) \neq \emptyset$ that $\left(x^{*}, y^{*}, z^{*}\right) \in \operatorname{Fix}(\mathcal{Q})$ and so $x^{*} \in \operatorname{Fix}\left(S_{1}\right), y^{*} \in \operatorname{Fix}\left(S_{2}\right)$ and $z^{*} \in \operatorname{Fix}\left(S_{3}\right)$. Hence, for each $n \in \mathbb{N}$, we can write

$$
\left\{\begin{array}{l}
x^{*}=\left(1-\alpha_{n}-\beta_{n}\right) x^{*}+\alpha_{n} S_{1}^{n} J_{\varphi_{1}}^{\rho}\left(g_{1}\left(y^{*}\right)-\rho T_{1}\left(y^{*}, z^{*}, x^{*}\right)\right)+\beta_{n} x^{*}, \\
y^{*}=\left(1-\alpha_{n}^{\prime}-\beta_{n}^{\prime}\right) y^{*}+\alpha_{n}^{\prime} S_{2}^{n} J_{\varphi_{2}}^{\eta}\left(g_{2}\left(z^{*}\right)-\eta T_{2}\left(z^{*}, x^{*}, y^{*}\right)\right)+\beta_{n}^{\prime} y^{*}, \\
z^{*}=\left(1-\alpha_{n}^{\prime \prime}-\beta_{n}^{\prime \prime}\right) z^{*}+\alpha_{n}^{\prime \prime} S_{3}^{n} J_{\varphi_{3}}^{\gamma}\left(g_{3}\left(x^{*}\right)-\gamma T_{3}\left(x^{*}, y^{*}, z^{*}\right)\right)+\beta_{n}^{\prime \prime} z^{*},
\end{array}\right.
$$


where the sequences $\left\{\alpha_{n}\right\}_{n=1}^{\infty},\left\{\alpha_{n}^{\prime}\right\}_{n=1}^{\infty},\left\{\alpha_{n}^{\prime \prime}\right\}_{n=1}^{\infty},\left\{\beta_{n}\right\}_{n=1}^{\infty},\left\{\beta_{n}^{\prime}\right\}_{n=1}^{\infty}$ and $\left\{\beta_{n}^{\prime \prime}\right\}_{n=1}^{\infty}$ are the same as in Algorithm 4.1. Let $\Gamma=\sup _{n \geq 1}\left\{\left\|j_{n}-x^{*}\right\|,\left\|q_{n}-y^{*}\right\|,\left\|t_{n}-z^{*}\right\|\right\}$. It follows from (4.3), (5.1) and the assumptions that

$$
\begin{aligned}
\| x_{n+1} & -x^{*} \| \\
\leq & \left(1-\alpha_{n}-\beta_{n}\right)\left\|x_{n}-x^{*}\right\|+\alpha_{n} \| S_{1}^{n} J_{\varphi_{1}}^{\rho}\left(g_{1}\left(y_{n+1}\right)-\rho T_{1}\left(y_{n+1}, z_{n+1}, x_{n}\right)\right) \\
& \quad-S_{1}^{n} J_{\varphi_{1}}^{\rho}\left(g_{1}\left(y^{*}\right)-\rho T_{1}\left(y^{*}, z^{*}, x^{*}\right)\right)\left\|+\beta_{n}\right\| j_{n}-x^{*}\left\|+\alpha_{n}\right\| e_{n}\|+\| r_{n} \| \\
\leq & \left(1-\alpha_{n}-\beta_{n}\right)\left\|x_{n}-x^{*}\right\| \\
& +\alpha_{n} L_{1}\left(\left\|g_{1}\left(y_{n+1}\right)-g_{1}\left(y^{*}\right)-\rho\left(T_{1}\left(y_{n+1}, z_{n+1}, x_{n}\right)-T_{1}\left(y^{*}, z^{*}, x^{*}\right)\right)\right\|+b_{n}\right) \\
& +\alpha_{n}\left(\left\|e_{n}^{\prime}\right\|+\left\|e_{n}^{\prime \prime}\right\|\right)+\left\|r_{n}\right\|+\beta_{n} \Gamma \\
\leq & \left(1-\alpha_{n}-\beta_{n}\right)\left\|x_{n}-x^{*}\right\|+\alpha_{n} L_{1}\left(\left\|y_{n+1}-y^{*}-\left(g_{1}\left(y_{n+1}\right)-g_{1}\left(y^{*}\right)\right)\right\|\right. \\
& \left.+\left\|y_{n+1}-y^{*}-\rho\left(T_{1}\left(y_{n+1}, z_{n+1}, x_{n}\right)-T_{1}\left(y^{*}, z^{*}, x^{*}\right)\right)\right\|+b_{n}\right) \\
& +\alpha_{n}\left\|e_{n}^{\prime}\right\|+\left\|e_{n}^{\prime \prime}\right\|+\left\|r_{n}\right\|+\beta_{n} \Gamma .
\end{aligned}
$$

Since $g_{1}$ is $\pi_{1}$-strongly monotone and $\delta_{1}$-Lipschitz continuous, and $T_{1}$ is $\varsigma_{1}$-strongly monotone and $\sigma_{1}$-Lipschitz continuous in the first variable, similar to the proofs of (3.7) and (3.8), one can prove that

$$
\left\|y_{n+1}-y^{*}-\left(g_{1}\left(y_{n+1}\right)-g_{1}\left(y^{*}\right)\right)\right\| \leq \sqrt{1-2 \pi_{1}+\delta_{1}^{2}}\left\|y_{n+1}-y^{*}\right\|
$$

and

$$
\begin{aligned}
& \left\|y_{n+1}-y^{*}-\rho\left(T_{1}\left(y_{n+1}, z_{n+1}, x_{n}\right)-T_{1}\left(y^{*}, z^{*}, x^{*}\right)\right)\right\| \\
& \quad \leq \sqrt{1-2 \rho \varsigma_{1}+\rho^{2} \sigma_{1}^{2}}\left\|y_{n+1}-y^{*}\right\| .
\end{aligned}
$$

Substituting (5.3) and (5.4) in (5.2), we get

$$
\begin{aligned}
\left\|x_{n+1}-x^{*}\right\| \leq & \left(1-\alpha_{n}-\beta_{n}\right)\left\|x_{n}-x^{*}\right\|+\alpha_{n} L_{1} \theta\left\|y_{n+1}-y^{*}\right\| \\
& +\alpha_{n} L_{1} b_{n}+\alpha_{n}\left\|e_{n}^{\prime}\right\|+\left\|e_{n}^{\prime \prime}\right\|+\left\|r_{n}\right\|+\beta_{n} \Gamma,
\end{aligned}
$$

where $\theta$ is the same as in (3.13). It follows from (4.3) and (5.1) that

$$
\begin{aligned}
&\left\|y_{n+1}-y^{*}\right\| \\
& \leq\left(1-\alpha_{n}^{\prime}-\beta_{n}^{\prime}\right)\left\|x_{n}-y^{*}\right\|+\alpha_{n}^{\prime} \| S_{2}^{n} J_{\varphi_{2}}^{\eta}\left(g_{2}\left(z_{n+1}\right)-\eta T_{2}\left(z_{n+1}, x_{n}, y_{n}\right)\right) \\
& \quad-S_{2}^{n} J_{\varphi_{2}}^{\eta}\left(g_{2}\left(z^{*}\right)-\eta T_{2}\left(z^{*}, x^{*}, y^{*}\right)\right)\left\|+\beta_{n}^{\prime}\right\| q_{n}-y^{*}\left\|+\alpha_{n}^{\prime}\right\| p_{n}\|+\| k_{n} \| \\
& \leq\left(1-\alpha_{n}^{\prime}-\beta_{n}^{\prime}\right)\left\|x_{n}-y^{*}\right\| \\
&+\alpha_{n}^{\prime} L_{2}\left(\left\|g_{2}\left(z_{n+1}\right)-g_{2}\left(z^{*}\right)-\eta\left(T_{2}\left(z_{n+1}, x_{n}, y_{n}\right)-T_{2}\left(z^{*}, x^{*}, y^{*}\right)\right)\right\|+c_{n}\right) \\
&+\alpha_{n}^{\prime}\left(\left\|p_{n}^{\prime}\right\|+\left\|p_{n}^{\prime \prime}\right\|\right)+\left\|k_{n}\right\|+\beta_{n}^{\prime} \Gamma \\
& \leq\left(1-\alpha_{n}^{\prime}-\beta_{n}^{\prime}\right)\left\|x_{n}-y^{*}\right\|+\alpha_{n}^{\prime} L_{2}\left(\left\|z_{n+1}-z^{*}-\left(g_{2}\left(z_{n+1}\right)-g_{2}\left(z^{*}\right)\right)\right\|\right.
\end{aligned}
$$




$$
\begin{aligned}
& \left.+\left\|z_{n+1}-z^{*}-\eta\left(T_{2}\left(z_{n+1}, x_{n}, y_{n}\right)-T_{2}\left(z^{*}, x^{*}, y^{*}\right)\right)\right\|+c_{n}\right) \\
& +\alpha_{n}^{\prime}\left\|p_{n}^{\prime}\right\|+\left\|p_{n}^{\prime \prime}\right\|+\left\|k_{n}\right\|+\beta_{n}^{\prime} \Gamma .
\end{aligned}
$$

Since $g_{2}$ is $\pi_{2}$-strongly monotone and $\delta_{2}$-Lipschitz continuous, and $T_{2}$ is $\varsigma_{2}$-strongly monotone and $\sigma_{2}$-Lipschitz continuous in the first variable, we can get

$$
\left\|z_{n+1}-z^{*}-\left(g_{2}\left(z_{n+1}\right)-g_{2}\left(z^{*}\right)\right)\right\| \leq \sqrt{1-2 \pi_{2}+\delta_{2}^{2}}\left\|z_{n+1}-z^{*}\right\|
$$

and

$$
\left\|z_{n+1}-z^{*}-\eta\left(T_{2}\left(z_{n+1}, x_{n}, y_{n}\right)-T_{2}\left(z^{*}, x^{*}, y^{*}\right)\right)\right\| \leq \sqrt{1-2 \eta \zeta_{2}+\eta^{2} \sigma_{2}^{2}}\left\|z_{n+1}-z^{*}\right\| .
$$

Combining (5.6)-(5.8), we conclude that

$$
\begin{aligned}
\left\|y_{n+1}-y^{*}\right\| \leq & \left(1-\alpha_{n}^{\prime}-\beta_{n}^{\prime}\right)\left\|x_{n}-y^{*}\right\|+\alpha_{n}^{\prime} L_{2} \varrho\left\|z_{n+1}-z^{*}\right\| \\
& +\alpha_{n}^{\prime} L_{2} c_{n}+\alpha_{n}^{\prime}\left\|p_{n}^{\prime}\right\|+\left\|p_{n}^{\prime \prime}\right\|+\left\|k_{n}\right\|+\beta_{n}^{\prime} \Gamma,
\end{aligned}
$$

where $\varrho$ is the same as in (3.13). From (4.3) and (5.1), it follows that

$$
\begin{aligned}
\| z_{n+1}- & z^{*} \| \\
\leq & \left(1-\alpha_{n}^{\prime \prime}-\beta_{n}^{\prime \prime}\right)\left\|x_{n}-z^{*}\right\|+\alpha_{n}^{\prime \prime} \| S_{3}^{n} J_{\varphi_{3}}^{\gamma}\left(g_{3}\left(x_{n}\right)-\gamma T_{3}\left(x_{n}, y_{n}, z_{n}\right)\right) \\
& -S_{3}^{n} J_{\varphi_{3}}^{\gamma}\left(g_{3}\left(x^{*}\right)-\gamma T_{3}\left(x^{*}, y^{*}, z^{*}\right)\right)\left\|+\beta_{n}^{\prime \prime}\right\| t_{n}-z^{*}\left\|+\alpha_{n}^{\prime \prime}\right\| s_{n}\|+\| l_{n} \| \\
\leq & \left(1-\alpha_{n}^{\prime \prime}-\beta_{n}^{\prime \prime}\right)\left\|x_{n}-z^{*}\right\|+\alpha_{n}^{\prime \prime} L_{3}\left(\| g_{3}\left(x_{n}\right)-g_{3}\left(x^{*}\right)\right. \\
& \left.-\gamma\left(T_{3}\left(x_{n}, y_{n}, z_{n}\right)-T_{3}\left(x^{*}, y^{*}, z^{*}\right)\right) \|+d_{n}\right)+\alpha_{n}^{\prime \prime}\left(\left\|s_{n}^{\prime}\right\|+\left\|s_{n}^{\prime \prime}\right\|\right)+\left\|l_{n}\right\|+\beta_{n}^{\prime \prime} \Gamma \\
\leq & \left(1-\alpha_{n}^{\prime \prime}-\beta_{n}^{\prime \prime}\right)\left\|x_{n}-z^{*}\right\|+\alpha_{n}^{\prime \prime} L_{3}\left(\left\|x_{n}-x^{*}-\left(g_{3}\left(x_{n}\right)-g_{3}\left(x^{*}\right)\right)\right\|\right. \\
& \left.+\left\|x_{n}-x^{*}-\gamma\left(T_{3}\left(x_{n}, y_{n}, z_{n}\right)-T_{3}\left(x^{*}, y^{*}, z^{*}\right)\right)\right\|+d_{n}\right) \\
& +\alpha_{n}^{\prime \prime}\left\|s_{n}^{\prime}\right\|+\left\|s_{n}^{\prime \prime}\right\|+\left\|l_{n}\right\|+\beta_{n}^{\prime \prime} \Gamma .
\end{aligned}
$$

Because $g_{3}$ is $\pi_{3}$-strongly monotone and $\delta_{3}$-Lipschitz continuous, and $T_{3}$ is $\zeta_{3}$-strongly monotone and $\sigma_{3}$-Lipschitz continuous in the first variable, we can obtain

$$
\left\|x_{n}-x^{*}-\left(g_{3}\left(x_{n}\right)-g_{3}\left(x^{*}\right)\right)\right\| \leq \sqrt{1-2 \pi_{3}+\delta_{3}^{2}}\left\|x_{n}-x^{*}\right\|
$$

and

$$
\left\|x_{n}-x^{*}-\gamma\left(T_{3}\left(x_{n}, y_{n}, z_{n}\right)-T_{3}\left(x^{*}, y^{*}, z^{*}\right)\right)\right\| \leq \sqrt{1-2 \gamma \varsigma_{3}+\gamma^{2} \sigma_{3}^{2}}\left\|x_{n}-x^{*}\right\| .
$$

Substituting (5.11) and (5.12) in (5.10), deduce that

$$
\begin{aligned}
\left\|z_{n+1}-z^{*}\right\| \leq & \left(1-\alpha_{n}^{\prime \prime}-\beta_{n}^{\prime \prime}\right)\left\|x_{n}-z^{*}\right\|+\alpha_{n}^{\prime \prime} L_{3} \vartheta\left\|x_{n}-x^{*}\right\| \\
& +\alpha_{n}^{\prime \prime} L_{3} d_{n}+\alpha_{n}^{\prime \prime}\left\|s_{n}^{\prime}\right\|+\left\|s_{n}^{\prime \prime}\right\|+\left\|l_{n}\right\|+\beta_{n}^{\prime \prime} \Gamma
\end{aligned}
$$

where $\vartheta$ is the same as in (3.13). 
By using (5.13) and the fact that $L_{3} \vartheta<1$, we have

$$
\begin{aligned}
\left\|z_{n+1}-z^{*}\right\| \leq & \left(1-\alpha_{n}^{\prime \prime}-\beta_{n}^{\prime \prime}\right)\left\|x_{n}-z^{*}\right\|+\alpha_{n}^{\prime \prime} L_{3} \vartheta\left\|x_{n}-x^{*}\right\| \\
& +\alpha_{n}^{\prime \prime} L_{3} d_{n}+\alpha_{n}^{\prime \prime}\left\|s_{n}^{\prime}\right\|+\left\|s_{n}^{\prime \prime}\right\|+\left\|l_{n}\right\|+\beta_{n}^{\prime \prime} \Gamma \\
\leq & \left(1-\alpha_{n}^{\prime \prime}-\beta_{n}^{\prime \prime}\right)\left\|x_{n}-x^{*}\right\|+\alpha_{n}^{\prime \prime} L_{3} \vartheta\left\|x_{n}-x^{*}\right\|+\alpha_{n}^{\prime \prime} L_{3} d_{n} \\
& +\left(1-\alpha_{n}^{\prime \prime}-\beta_{n}^{\prime \prime}\right)\left\|x^{*}-z^{*}\right\|+\alpha_{n}^{\prime \prime}\left\|s_{n}^{\prime}\right\|+\left\|s_{n}^{\prime \prime}\right\|+\left\|l_{n}\right\|+\beta_{n}^{\prime \prime} \Gamma \\
\leq & \left(1-\alpha_{n}^{\prime \prime}-\beta_{n}^{\prime \prime}\right)\left\|x_{n}-x^{*}\right\|+\alpha_{n}^{\prime \prime}\left\|x_{n}-x^{*}\right\|+\alpha_{n}^{\prime \prime} L_{3} d_{n} \\
& +\left(1-\alpha_{n}^{\prime \prime}-\beta_{n}^{\prime \prime}\right)\left\|x^{*}-z^{*}\right\|+\alpha_{n}^{\prime \prime}\left\|s_{n}^{\prime}\right\|+\left\|s_{n}^{\prime \prime}\right\|+\left\|l_{n}\right\|+\beta_{n}^{\prime \prime} \Gamma \\
\leq & \left\|x_{n}-x^{*}\right\|+\alpha_{n}^{\prime \prime} L_{3} d_{n}+\left(1-\alpha_{n}^{\prime \prime}-\beta_{n}^{\prime \prime}\right)\left\|x^{*}-z^{*}\right\| \\
& +\alpha_{n}^{\prime \prime}\left\|s_{n}^{\prime}\right\|+\left\|s_{n}^{\prime \prime}\right\|+\left\|l_{n}\right\|+\beta_{n}^{\prime \prime} \Gamma .
\end{aligned}
$$

It follows from (5.9), (5.14) and the fact that $L_{2} \varrho<1$ that

$$
\begin{aligned}
\left\|y_{n+1}-y^{*}\right\| \leq & \left(1-\alpha_{n}^{\prime}-\beta_{n}^{\prime}\right)\left\|x_{n}-y^{*}\right\|+\alpha_{n}^{\prime} L_{2} \varrho\left\|z_{n+1}-z^{*}\right\| \\
& +\alpha_{n}^{\prime} L_{2} c_{n}+\alpha_{n}^{\prime}\left\|p_{n}^{\prime}\right\|+\left\|p_{n}^{\prime \prime}\right\|+\left\|k_{n}\right\|+\beta_{n}^{\prime} \Gamma \\
\leq & \left(1-\alpha_{n}^{\prime}-\beta_{n}^{\prime}\right)\left\|x_{n}-x^{*}\right\|+\left(1-\alpha_{n}^{\prime}-\beta_{n}^{\prime}\right)\left\|x^{*}-y^{*}\right\| \\
& +\alpha_{n}^{\prime} L_{2} \varrho\left\|z_{n+1}-z^{*}\right\|+\alpha_{n}^{\prime} L_{2} c_{n}+\alpha_{n}^{\prime}\left\|p_{n}^{\prime}\right\|+\left\|p_{n}^{\prime \prime}\right\|+\left\|k_{n}\right\|+\beta_{n}^{\prime} \Gamma \\
\leq & \left(1-\alpha_{n}^{\prime}-\beta_{n}^{\prime}\right)\left\|x_{n}-x^{*}\right\|+\left(1-\alpha_{n}^{\prime}-\beta_{n}^{\prime}\right)\left\|x^{*}-y^{*}\right\| \\
& +\alpha_{n}^{\prime} L_{2} \varrho\left(\left\|x_{n}-x^{*}\right\|+\alpha_{n}^{\prime \prime} L_{3} d_{n}+\left(1-\alpha_{n}^{\prime \prime}-\beta_{n}^{\prime \prime}\right)\left\|x^{*}-z^{*}\right\|\right. \\
& \left.+\alpha_{n}^{\prime \prime}\left\|s_{n}^{\prime}\right\|+\left\|s_{n}^{\prime \prime}\right\|+\left\|l_{n}\right\|+\beta_{n}^{\prime \prime} \Gamma\right)+\alpha_{n}^{\prime} L_{2} c_{n}+\alpha_{n}^{\prime}\left\|p_{n}^{\prime}\right\|+\left\|p_{n}^{\prime \prime}\right\|+\left\|k_{n}\right\|+\beta_{n}^{\prime} \Gamma \\
\leq & \left\|x_{n}-x^{*}\right\|+\left(1-\alpha_{n}^{\prime}-\beta_{n}^{\prime}\right)\left\|x^{*}-y^{*}\right\|+\alpha_{n}^{\prime}\left(1-\alpha_{n}^{\prime \prime}-\beta_{n}^{\prime \prime}\right) L_{2} \varrho\left\|x^{*}-z^{*}\right\| \\
& +\alpha_{n}^{\prime} \alpha_{n}^{\prime \prime} L_{2} L_{3} \varrho d_{n}+\alpha_{n}^{\prime} \alpha_{n}^{\prime \prime} L_{2} \varrho\left\|s_{n}^{\prime}\right\|+\alpha_{n}^{\prime} L_{2} \varrho\left\|s_{n}^{\prime \prime}\right\|+\alpha_{n}^{\prime} L_{2} \varrho\left\|l_{n}\right\|+\alpha_{n}^{\prime} L_{2} \varrho \beta_{n}^{\prime \prime} \Gamma \\
& +\alpha_{n}^{\prime} L_{2} c_{n}+\alpha_{n}^{\prime}\left\|p_{n}^{\prime}\right\|+\left\|p_{n}^{\prime \prime}\right\|+\left\|k_{n}\right\|+\beta_{n}^{\prime} \Gamma .
\end{aligned}
$$

Applying (5.5) and (5.15), it follows that

$$
\begin{aligned}
\| x_{n+1} & -x^{*} \| \\
\leq & \left(1-\alpha_{n}-\beta_{n}\right)\left\|x_{n}-x^{*}\right\|+\alpha_{n} L_{1} \theta\left\|y_{n+1}-y^{*}\right\| \\
& +\alpha_{n} L_{1} b_{n}+\alpha_{n}\left\|e_{n}^{\prime}\right\|+\left\|e_{n}^{\prime \prime}\right\|+\left\|r_{n}\right\|+\beta_{n} \Gamma \\
\leq & \left(1-\alpha_{n}-\beta_{n}\right)\left\|x_{n}-x^{*}\right\|+\alpha_{n} L_{1} \theta\left(\left\|x_{n}-x^{*}\right\|+\left(1-\alpha_{n}^{\prime}-\beta_{n}^{\prime}\right)\left\|x^{*}-y^{*}\right\|\right. \\
& +\alpha_{n}^{\prime}\left(1-\alpha_{n}^{\prime \prime}-\beta_{n}^{\prime \prime}\right) L_{2} \varrho\left\|x^{*}-z^{*}\right\|+\alpha_{n}^{\prime} \alpha_{n}^{\prime \prime} L_{2} L_{3} \varrho d_{n}+\alpha_{n}^{\prime} \alpha_{n}^{\prime \prime} L_{2} \varrho\left\|s_{n}^{\prime}\right\|+\alpha_{n}^{\prime} L_{2} \varrho\left\|s_{n}^{\prime \prime}\right\| \\
& \left.+\alpha_{n}^{\prime} L_{2} \varrho\left\|l_{n}\right\|+\alpha_{n}^{\prime} L_{2} \varrho \beta_{n}^{\prime \prime} \Gamma+\alpha_{n}^{\prime} L_{2} c_{n}+\alpha_{n}^{\prime}\left\|p_{n}^{\prime}\right\|+\left\|p_{n}^{\prime \prime}\right\|+\left\|k_{n}\right\|+\beta_{n}^{\prime} \Gamma\right) \\
& +\alpha_{n} L_{1} b_{n}+\alpha_{n}\left\|e_{n}^{\prime}\right\|+\left\|e_{n}^{\prime \prime}\right\|+\left\|r_{n}\right\|+\beta_{n} \Gamma \\
\leq & \left(1-\alpha_{n}-\beta_{n}\right)\left\|x_{n}-x^{*}\right\|+\alpha_{n} L_{1} \theta\left\|x_{n}-x^{*}\right\|+\alpha_{n}\left(1-\alpha_{n}^{\prime}-\beta_{n}^{\prime}\right) L_{1} \theta\left\|x^{*}-y^{*}\right\| \\
& +\alpha_{n} \alpha_{n}^{\prime}\left(1-\alpha_{n}^{\prime \prime}-\beta_{n}^{\prime \prime}\right) L_{1} L_{2} \theta \varrho\left\|x^{*}-z^{*}\right\|+\alpha_{n} \alpha_{n}^{\prime} \alpha_{n}^{\prime \prime} L_{1} L_{2} L_{3} \theta \varrho d_{n}+\alpha_{n} \alpha_{n}^{\prime} L_{1} L_{2} \theta c_{n} \\
& +\alpha_{n} L_{1} b_{n}+\alpha_{n} \alpha_{n}^{\prime} \alpha_{n}^{\prime \prime} L_{1} L_{2} \theta \varrho\left\|s_{n}^{\prime}\right\|+\alpha_{n} \alpha_{n}^{\prime} L_{1} L_{2} \theta \varrho\left\|s_{n}^{\prime \prime}\right\|+\alpha_{n} \alpha_{n}^{\prime} L_{1} L_{2} \theta \varrho\left\|l_{n}\right\|
\end{aligned}
$$




$$
\begin{aligned}
& +\alpha_{n} \alpha_{n}^{\prime} L_{1} \theta\left\|p_{n}^{\prime}\right\|+\alpha_{n} L_{1} \theta\left\|p_{n}^{\prime \prime}\right\|+\alpha_{n} L_{1} \theta\left\|k_{n}\right\|+\alpha_{n}\left\|e_{n}^{\prime}\right\| \\
& +\left\|e_{n}^{\prime \prime}\right\|+\left\|r_{n}\right\|+\left(\alpha_{n} \alpha_{n}^{\prime} L_{1} L_{2} \theta \varrho \beta_{n}^{\prime \prime}+\alpha_{n} L_{1} \theta \beta_{n}^{\prime}+\beta_{n}\right) \Gamma \\
\leq & \left(1-\alpha_{n}\left(1-L_{1} \theta\right)\right)\left\|x_{n}-x^{*}\right\| \\
& +\alpha_{n}\left(1-L_{1} \theta\right)\left[\frac{\left(1-\alpha_{n}^{\prime}-\beta_{n}^{\prime}\right) L_{1} \theta\left\|x^{*}-y^{*}\right\|+\alpha_{n}^{\prime}\left(1-\alpha_{n}^{\prime \prime}-\beta_{n}^{\prime \prime}\right) L_{1} L_{2} \theta \varrho\left\|x^{*}-z^{*}\right\|}{1-L_{1} \theta}\right. \\
& \left.+\frac{\alpha_{n}^{\prime} \alpha_{n}^{\prime \prime} L_{1} L_{2} L_{3} \theta \varrho d_{n}+\alpha_{n}^{\prime} L_{1} L_{2} \theta c_{n}+L_{1} b_{n}+\alpha_{n}^{\prime} \alpha_{n}^{\prime \prime} L_{1} L_{2} \theta \varrho\left\|s_{n}^{\prime}\right\|+\alpha_{n}^{\prime} L_{1} \theta\left\|p_{n}^{\prime}\right\|+\left\|e_{n}^{\prime}\right\|}{1-L_{1} \theta}\right] \\
& +\alpha_{n} \alpha_{n}^{\prime} L_{1} L_{2} \theta \varrho\left\|s_{n}^{\prime \prime}\right\|+\alpha_{n} \alpha_{n}^{\prime} L_{1} L_{2} \theta \varrho\left\|l_{n}\right\|+\alpha_{n} L_{1} \theta\left\|p_{n}^{\prime \prime}\right\|+\alpha_{n} L_{1} \theta\left\|k_{n}\right\| \\
& +\left\|e_{n}^{\prime \prime}\right\|+\left\|r_{n}\right\|+\left(\alpha_{n} \alpha_{n}^{\prime} L_{1} L_{2} \theta \varrho \beta_{n}^{\prime \prime}+\alpha_{n} L_{1} \theta \beta_{n}^{\prime}+\beta_{n}\right) \Gamma .
\end{aligned}
$$

From $\sum_{n=1}^{\infty} \beta_{n}<\infty, \sum_{n=1}^{\infty} \beta_{n}^{\prime}<\infty$ and $\sum_{n=1}^{\infty} \beta_{n}^{\prime \prime}<\infty$, we infer that $\lim _{n \rightarrow \infty} \beta_{n}=\lim _{n \rightarrow \infty} \beta_{n}^{\prime}=$ $\lim _{n \rightarrow \infty} \beta_{n}^{\prime \prime}=0$. Since $L_{1} \theta<1, \lim _{n \rightarrow \infty} \alpha_{n}^{\prime}=\lim _{n \rightarrow \infty} \alpha_{n}^{\prime \prime}=1$ and $\lim _{n \rightarrow \infty} b_{n}=\lim _{n \rightarrow \infty} c_{n}=$ $\lim _{n \rightarrow \infty} d_{n}=0$, in view of (4.4), it is evident that the conditions of Lemma 5.1 are satisfied and so Lemma 5.1 and (5.16) guarantee that $x_{n} \rightarrow x^{*}$, as $n \rightarrow \infty$. Because $\sum_{n=1}^{\infty}\left\|l_{n}\right\|<\infty$, $\sum_{n=1}^{\infty}\left\|k_{n}\right\|<\infty, \sum_{n=1}^{\infty}\left\|s_{n}^{\prime \prime}\right\|<\infty$ and $\sum_{n=1}^{\infty}\left\|p_{n}^{\prime \prime}\right\|<\infty$, we have $\left\|l_{n}\right\| \rightarrow 0,\left\|k_{n}\right\| \rightarrow 0$, $\left\|s_{n}^{\prime \prime}\right\| \rightarrow 0$ and $\left\|p_{n}^{\prime \prime}\right\| \rightarrow 0$, as $n \rightarrow \infty$. Now, it follows from (4.4), (5.14) and (5.15) that $y_{n} \rightarrow y^{*}$ and $z_{n} \rightarrow z^{*}$, as $n \rightarrow \infty$. Therefore, the sequence $\left\{\left(x_{n}, y_{n}, z_{n}\right)\right\}_{n=1}^{\infty}$ generated by Algorithm 4.1 converges strongly to the unique solution $\left(x^{*}, y^{*}, z^{*}\right)$ of SGNMVID (2.1), that is, the only element of $\operatorname{Fix}(\mathcal{Q}) \cap \operatorname{SGNMVID}\left(\mathcal{H}, T_{i}, g_{i}, \varphi_{i}, i=1,2,3\right)$. This completes the proof.

Theorem 5.3 Suppose that $T_{i}, g_{i}, \varphi_{i}(i=1,2,3), \rho, \eta$ and $\gamma$ are the same as in Theorem 3.2 and let all the conditions of Theorem 3.2 hold. Then the iterative sequence $\left\{\left(x_{n}, y_{n}, z_{n}\right)\right\}_{n=1}^{\infty}$ generated by Algorithm 4.2 converges strongly to the unique solution of SGNMVID (2.1).

Theorem 5.4 Let $T_{i}, g_{i}, \varphi_{i}, S_{i}(i=1,2,3), \mathcal{Q}, \rho, \eta$ and $\gamma$ be the same as in Theorem 5.2 and let all the conditions of Theorem 5.2 hold. Then the iterative sequence $\left\{\left(x_{n}, y_{n}, z_{n}\right)\right\}_{n=1}^{\infty}$ generated by Algorithm 4.4 converges strongly to the only element of $\operatorname{Fix}(\mathcal{Q}) \cap$ $\operatorname{SGNMVID}\left(\mathcal{H}, T_{i}, g_{i}, \varphi_{i}, i=1,2,3\right)$.

Proof Theorem 3.2 guarantees the existence of a unique solution $\left(x^{*}, y^{*}, z^{*}\right) \in \mathcal{H} \times \mathcal{H} \times \mathcal{H}$ for SGNMVID (2.1). Hence, Lemma 3.1 implies that $x^{*}=J_{\varphi_{1}}^{\rho}\left(g_{1}\left(y^{*}\right)-\rho T_{1}\left(y^{*}, z^{*}, x^{*}\right)\right), y^{*}=$ $J_{\varphi_{2}}^{\eta}\left(g_{2}\left(z^{*}\right)-\eta T_{2}\left(z^{*}, x^{*}, y^{*}\right)\right), z^{*}=J_{\varphi_{3}}^{\gamma}\left(g_{3}\left(x^{*}\right)-\gamma T_{3}\left(x^{*}, y^{*}, z^{*}\right)\right)$. Since $\operatorname{SGNMVID}\left(\mathcal{H}, T_{i}, g_{i}, \varphi_{i}\right.$, $i=1,2,3)$ is a singleton set, by using $\operatorname{Fix}(\mathcal{Q}) \cap \operatorname{SGNMVID}\left(\mathcal{H}, T_{i}, g_{i}, \varphi_{i}, i=1,2,3\right) \neq \emptyset$, we conclude that $\left(x^{*}, y^{*}, z^{*}\right) \in \operatorname{Fix}(\mathcal{Q})$ and $\operatorname{so} x^{*} \in \operatorname{Fix}\left(S_{1}\right), y^{*} \in \operatorname{Fix}\left(S_{2}\right)$ and $z^{*} \in \operatorname{Fix}\left(S_{3}\right)$. Hence, in view of Remark 4.3, for each $n \in \mathbb{N}$, we can write

$$
\left\{\begin{array}{l}
x^{*}=S_{1}^{n} J_{\varphi_{1}}^{\rho}(u), \quad y^{*}=S_{2}^{n} J_{\varphi_{2}}^{\eta}(v), \quad z^{*}=S_{3}^{n} J_{\varphi_{3}}^{\gamma}(w), \\
u=\left(1-\alpha_{n}-\beta_{n}\right) u+\alpha_{n}\left(g_{1}\left(y^{*}\right)-\rho T_{1}\left(y^{*}, z^{*}, x^{*}\right)\right)+\beta_{n} u, \\
v=\left(1-\alpha_{n}-\beta_{n}\right) v+\alpha_{n}\left(g_{2}\left(z^{*}\right)-\eta T_{2}\left(z^{*}, x^{*}, y^{*}\right)\right)+\beta_{n} v, \\
w=\left(1-\alpha_{n}-\beta_{n}\right) w+\alpha_{n}\left(g_{3}\left(x^{*}\right)-\gamma T_{3}\left(x^{*}, y^{*}, z^{*}\right)\right)+\beta_{n} w,
\end{array}\right.
$$


where the sequences $\left\{\alpha_{n}\right\}_{n=1}^{\infty}$ and $\left\{\beta_{n}\right\}_{n=1}^{\infty}$ are the same as in Algorithm 4.4. Let $\hat{\Gamma}=$ $\sup _{n \geq 1}\left\{\left\|j_{n}-u\right\|,\left\|q_{n}-v\right\|,\left\|t_{n}-w\right\|\right\}$. By using (4.6), (5.17) and the assumptions, we have

$$
\begin{aligned}
& \left\|u_{n+1}-u\right\| \\
& \leq\left(1-\alpha_{n}-\beta_{n}\right)\left\|u_{n}-u\right\|+\alpha_{n}\left\|g_{1}\left(y_{n}\right)-g_{1}\left(y^{*}\right)-\rho\left(T_{1}\left(y_{n}, z_{n}, x_{n}\right)-T_{1}\left(y^{*}, z^{*}, x^{*}\right)\right)\right\| \\
& +\beta_{n}\left\|j_{n}-u\right\|+\alpha_{n}\left(\left\|e_{n}^{\prime}\right\|+\left\|e_{n}^{\prime \prime}\right\|\right)+\left\|r_{n}\right\| \\
& \leq\left(1-\alpha_{n}-\beta_{n}\right)\left\|u_{n}-u\right\|+\alpha_{n}\left\|y_{n}-y^{*}-\left(g_{1}\left(y_{n}\right)-g_{1}\left(y^{*}\right)\right)\right\| \\
& +\alpha_{n}\left\|y_{n}-y^{*}-\rho\left(T_{1}\left(y_{n}, z_{n}, x_{n}\right)-T_{1}\left(y^{*}, z^{*}, x^{*}\right)\right)\right\| \\
& +\alpha_{n}\left\|e_{n}^{\prime}\right\|+\left\|e_{n}^{\prime \prime}\right\|+\left\|r_{n}\right\|+\beta_{n} \hat{\Gamma} \text {. }
\end{aligned}
$$

Since $g_{1}$ is $\pi_{1}$-strongly monotone and $\delta_{1}$-Lipschitz continuous, and $T_{1}$ is $\varsigma_{1}$-strongly monotone and $\sigma_{1}$-Lipschitz continuous in the first variable, similar to the proofs of (3.7) and (3.8), one can prove that

$$
\left\|y_{n}-y^{*}-\left(g_{1}\left(y_{n}\right)-g_{1}\left(y^{*}\right)\right)\right\| \leq \sqrt{1-2 \pi_{1}+\delta_{1}^{2}}\left\|y_{n}-y^{*}\right\|
$$

and

$$
\left\|y_{n}-y^{*}-\rho\left(T_{1}\left(y_{n}, z_{n}, x_{n}\right)-T_{1}\left(y^{*}, z^{*}, x^{*}\right)\right)\right\| \leq \sqrt{1-2 \rho \varsigma_{1}+\rho^{2} \sigma_{1}^{2}}\left\|y_{n}-y^{*}\right\| .
$$

Combining (5.18)-(5.20), we get

$$
\begin{aligned}
\left\|u_{n+1}-u\right\| \leq & \left(1-\alpha_{n}-\beta_{n}\right)\left\|u_{n}-u\right\|+\alpha_{n} \theta\left\|y_{n}-y^{*}\right\| \\
& +\alpha_{n}\left\|e_{n}^{\prime}\right\|+\left\|e_{n}^{\prime \prime}\right\|+\left\|r_{n}\right\|+\beta_{n} \hat{\Gamma},
\end{aligned}
$$

where $\theta$ is the same as in (3.13). It follows from (4.6) and (5.17) that

$$
\begin{aligned}
\left\|y_{n}-y^{*}\right\| & =\left\|S_{2}^{n} J_{\varphi_{2}}^{\eta}\left(v_{n}\right)-S_{2}^{n} J_{\varphi_{2}}^{\eta}(v)\right\| \leq L_{2}\left(\left\|J_{\varphi_{2}}^{\eta}\left(v_{n}\right)-J_{\varphi_{2}}^{\eta}(v)\right\|+c_{n}\right) \\
& \leq L_{2}\left(\left\|v_{n}-v\right\|+c_{n}\right) .
\end{aligned}
$$

Substituting (5.22) in (5.21), conclude that

$$
\begin{aligned}
\left\|u_{n+1}-u\right\| \leq & \left(1-\alpha_{n}-\beta_{n}\right)\left\|u_{n}-u\right\|+\alpha_{n} L_{2} \theta\left\|v_{n}-v\right\|+\alpha_{n} L_{2} \theta c_{n} \\
& +\alpha_{n}\left\|e_{n}^{\prime}\right\|+\left\|e_{n}^{\prime \prime}\right\|+\left\|r_{n}\right\|+\beta_{n} \hat{\Gamma} .
\end{aligned}
$$

Like in the proofs of (5.18)-(5.23), we can verify that

$$
\begin{aligned}
\left\|v_{n+1}-v\right\| \leq & \left(1-\alpha_{n}-\beta_{n}\right)\left\|v_{n}-v\right\|+\alpha_{n} L_{3} \varrho\left\|w_{n}-w\right\|+\alpha_{n} L_{3} \varrho d_{n} \\
& +\alpha_{n}\left\|p_{n}^{\prime}\right\|+\left\|p_{n}^{\prime \prime}\right\|+\left\|k_{n}\right\|+\beta_{n} \hat{\Gamma}
\end{aligned}
$$

and

$$
\begin{aligned}
\left\|w_{n+1}-w\right\| \leq & \left(1-\alpha_{n}-\beta_{n}\right)\left\|w_{n}-w\right\|+\alpha_{n} L_{1} \vartheta\left\|u_{n}-u\right\|+\alpha_{n} L_{1} \vartheta b_{n} \\
& +\alpha_{n}\left\|s_{n}^{\prime}\right\|+\left\|s_{n}^{\prime \prime}\right\|+\left\|l_{n}\right\|+\beta_{n} \hat{\Gamma},
\end{aligned}
$$

where $\varrho$ and $\vartheta$ are the same as in (3.13). 
Let $L=\max \left\{L_{i}: i=1,2,3\right\}$. Then, applying (5.23)-(5.25), we obtain

$$
\begin{aligned}
& \left\|\left(u_{n+1}, v_{n+1}, w_{n+1}\right)-(u, v, w)\right\|_{*} \\
& \leq\left(1-\alpha_{n}-\beta_{n}\right)\left\|\left(u_{n}, v_{n}, w_{n}\right)-(u, v, w)\right\|_{*} \\
& +\alpha_{n} L \lambda\left\|\left(u_{n}, v_{n}, w_{n}\right)-(u, v, w)\right\|_{*}+\alpha_{n} L \lambda\left(b_{n}+c_{n}+d_{n}\right) \\
& +\alpha_{n}\left\|\left(e_{n}^{\prime}, p_{n}^{\prime}, s_{n}^{\prime}\right)\right\|_{*}+\left\|\left(e_{n}^{\prime \prime}, p_{n}^{\prime \prime}, s_{n}^{\prime \prime}\right)\right\|_{*}+\left\|\left(r_{n}, k_{n}, l_{n}\right)\right\|_{*}+3 \beta_{n} \hat{\Gamma} \\
& \leq\left(1-\alpha_{n}(1-L \lambda)\right)\left\|\left(u_{n}, v_{n}, w_{n}\right)-(u, v, w)\right\|_{*} \\
& +\alpha_{n}(1-L \lambda) \frac{\left\|\left(e_{n}^{\prime}, p_{n}^{\prime}, s_{n}^{\prime}\right)\right\|_{*}+L \lambda\left(b_{n}+c_{n}+d_{n}\right)}{1-L \lambda} \\
& +\left\|\left(e_{n}^{\prime \prime}, p_{n}^{\prime \prime}, s_{n}^{\prime \prime}\right)\right\|_{*}+\left\|\left(r_{n}, k_{n}, l_{n}\right)\right\|_{*}+3 \beta_{n} \hat{\Gamma},
\end{aligned}
$$

where $\lambda$ is the same as in (3.14). Since $\sum_{n=1}^{\infty} \alpha_{n}=\infty, \sum_{n=1}^{\infty} \beta_{n}<\infty, L \lambda<1$ and $\lim _{n \rightarrow \infty} b_{n}=$ $\lim _{n \rightarrow \infty} c_{n}=\lim _{n \rightarrow \infty} d_{n}=0$, in view of (4.4), we note that all the conditions of Lemma 5.1 are satisfied. Hence, Lemma 5.1 and (5.26) guarantee that $\left(u_{n}, v_{n}, w_{n}\right) \rightarrow(u, v, w)$, as $n \rightarrow$ $\infty$. By using (4.6) and (5.17), we have

$$
\begin{aligned}
\left\|x_{n}-x^{*}\right\| & =\left\|S_{1}^{n} J_{\varphi_{1}}^{\rho}\left(u_{n}\right)-S_{1}^{n} J_{\varphi_{1}}^{\rho}(u)\right\| \\
& \leq L_{1}\left(\left\|J_{\varphi_{1}}^{\rho}\left(u_{n}\right)-J_{\varphi_{1}}^{\rho}(u)\right\|+b_{n}\right) \\
& \leq L_{1}\left(\left\|u_{n}-u\right\|+b_{n}\right)
\end{aligned}
$$

and

$$
\begin{aligned}
\left\|z_{n}-z^{*}\right\| & =\left\|S_{3}^{n} J_{\varphi_{3}}^{\gamma}\left(w_{n}\right)-S_{3}^{n} J_{\varphi_{3}}^{\gamma}(w)\right\| \\
& \leq L_{3}\left(\left\|J_{\varphi_{3}}^{\gamma}\left(w_{n}\right)-J_{\varphi_{3}}^{\gamma}(w)\right\|+d_{n}\right) \\
& \leq L_{3}\left(\left\|w_{n}-w\right\|+d_{n}\right) .
\end{aligned}
$$

Since $\lim _{n \rightarrow \infty} u_{n}=u, \lim _{n \rightarrow \infty} v_{n}=v, \lim _{n \rightarrow \infty} w_{n}=w$ and $\lim _{n \rightarrow \infty} b_{n}=\lim _{n \rightarrow \infty} c_{n}=$ $\lim _{n \rightarrow \infty} d_{n}=0$, from inequalities (5.22), (5.27) and (5.28) it follows that $y_{n} \rightarrow y^{*}, x_{n} \rightarrow x^{*}$ and $z_{n} \rightarrow z^{*}$, as $n \rightarrow \infty$. Hence, the sequence $\left\{\left(x_{n}, y_{n}, z_{n}\right)\right\}_{n=1}^{\infty}$ generated by Algorithm 4.4 converges strongly to the unique solution $\left(x^{*}, y^{*}, z^{*}\right)$ of SGNMVID (2.1), that is, the only element of $\operatorname{Fix}(\mathcal{Q}) \cap \operatorname{SGNMVID}\left(\mathcal{H}, T_{i}, g_{i}, \varphi_{i}, i=1,2,3\right)$. This completes the proof.

Like in the proof of Theorem 5.4, one can prove the convergence of the iterative sequences generated by Algorithms 4.5 and 4.6, and we omit their proofs.

Theorem 5.5 Suppose that $T_{i}, g_{i}, \varphi_{i}, S_{i}(i=1,2,3), \mathcal{Q}, \rho, \eta$ and $\gamma$ are the same as in Theorem 5.2 and let all the conditions of Theorem 5.2 hold. Then the iterative sequence $\left\{\left(x_{n}, y_{n}, z_{n}\right)\right\}_{n=1}^{\infty}$ generated by Algorithm 4.5 converges strongly to the only element of $\operatorname{Fix}(\mathcal{Q}) \cap \operatorname{SGNMVID}\left(\mathcal{H}, T_{i}, g_{i}, \varphi_{i}, i=1,2,3\right)$.

Theorem 5.6 Assume that $T_{i}, g_{i}, \varphi_{i}(i=1,2,3), \rho, \eta$ and $\gamma$ are the same as in Theorem 3.2 and let all the conditions of Theorem 3.2 hold. Then the iterative sequence $\left\{\left(x_{n}, y_{n}, z_{n}\right)\right\}_{n=1}^{\infty}$ generated by Algorithm 4.6 converges strongly to the unique solution of SGNMVID (2.1). 


\section{An important remark on a relaxed cocoercive mapping}

In view of Definition 3.1, we note that the relaxed cocoercivity condition is weaker than the strong monotonicity condition. In other words, the class of relaxed cocoercive mappings is more general than the class of strongly monotone mappings. However, it is worth to point out that if the considered mapping $T$ is $(\kappa, \theta)$-relaxed cocoercive and $\gamma$-Lipschitz mapping such that $\theta>\kappa \gamma^{2}$, then it must be a $\left(\theta-\kappa \gamma^{2}\right)$-strongly monotone mapping. Hence, the results that appeared in this paper can be also applied to a class of relaxed cocoercive mappings. In fact, one may rewrite the results considered under relaxed cocoercivity and Lipschitzian conditions of mappings and apply a known result on the strongly monotone condition to a new form. Below, we present an example of the mentioned situation.

For given three different nonlinear operators $T_{1}, T_{2}, g: \mathcal{H} \times \mathcal{H} \rightarrow \mathcal{H}$ and a continuous function $\varphi: \mathcal{H} \rightarrow \mathbb{R} \cup\{+\infty\}$, Noor [13] introduced and considered the problem of finding $\left(x^{*}, y^{*}\right) \in \mathcal{H} \times \mathcal{H}$ such that

$$
\begin{cases}\left\langle\rho T_{1}\left(y^{*}, x^{*}\right)+x^{*}-g\left(y^{*}\right), g(x)-x^{*}\right\rangle \geq \rho \varphi\left(x^{*}\right)-\rho \varphi(g(x)), & \forall x \in \mathcal{H} \\ \left\langle\eta T_{2}\left(x^{*}, y^{*}\right)+y^{*}-g\left(x^{*}\right), g(x)-y^{*}\right\rangle \geq \eta \varphi\left(y^{*}\right)-\eta \varphi(g(x)), & \forall x \in \mathcal{H}\end{cases}
$$

which is called a system of general mixed variational inequalities involving three different nonlinear operators (SGMVID). He also considered some spacial cases of SGMVID (6.1).

He proposed the following two-step iterative algorithm and its special forms for solving SGMVID (6.1) and studied the convergence analysis of the proposed iterative algorithms under certain conditions.

Algorithm 6.1 ([13], Algorithm 3.1) For arbitrary chosen initial points $x_{0}, y_{0} \in \mathcal{H}$, compute the sequences $\left\{x_{n}\right\}$ and $\left\{y_{n}\right\}$ by

$$
\begin{aligned}
& x_{n+1}=\left(1-a_{n}\right) x_{n}+a_{n} J_{\varphi}\left[g\left(y_{n}\right)-\rho T_{1}\left(y_{n}, x_{n}\right)\right], \\
& y_{n+1}=J_{\varphi}\left[g\left(x_{n+1}\right)-\eta T_{2}\left(x_{n+1}, y_{n}\right)\right],
\end{aligned}
$$

where $a_{n} \in[0,1]$ for all $n \geq 0$.

Theorem 6.2 ([13], Theorem 3.1) Let $x^{*}, y^{*}$ be the solution of SGMVID (6.1). Suppose that $T_{1}: \mathcal{H} \times \mathcal{H} \rightarrow \mathcal{H}$ is relaxed $\left(\gamma_{1}, r_{1}\right)$-cocoercive and $\mu_{1}$-Lipschitzian in the first variable, and $T_{2}: \mathcal{H} \times \mathcal{H} \rightarrow \mathcal{H}$ is relaxed $\left(\gamma_{2}, r_{2}\right)$-cocoercive and $\mu_{2}$-Lipschitzian in the first variable. Let $g$ be a relaxed $\left(\gamma_{3}, r_{3}\right)$-cocoercive and $\mu_{3}$-Lipschitzian. If

$$
\begin{aligned}
& \left|\rho-\frac{r_{1}-\gamma_{1} \mu_{1}^{2}}{\mu_{1}^{2}}\right|<\frac{\sqrt{\left(r_{1}-\gamma_{1} \mu_{1}^{2}\right)^{2}-\mu_{1}^{2} k(2-k)}}{\mu_{1}^{2}}, \\
& r_{1}>\gamma_{1} \mu_{1}^{2}+\mu_{1} \sqrt{k(2-k)}, \quad k<1, \\
& \left|\eta-\frac{r_{2}-\gamma_{2} \mu_{2}^{2}}{\mu_{2}^{2}}\right|<\frac{\sqrt{\left(r_{2}-\gamma_{2} \mu_{2}^{2}\right)^{2}-\mu_{2}^{2} k(2-k)}}{\mu_{2}^{2}}, \\
& r_{2}>\gamma_{2} \mu_{2}^{2}+\mu_{2} \sqrt{k(2-k)}, \quad k<1,
\end{aligned}
$$

where

$$
k=\sqrt{1-2\left(r_{3}-\gamma_{3} \mu_{3}^{2}\right)+\mu_{3}^{2}}
$$


and $a_{n} \in[0,1], \sum_{n=0}^{\infty} a_{n}=\infty$, then for arbitrarily chosen initial points $x_{0}, y_{0} \in \mathcal{H}, x_{n}$ and $y_{n}$ obtained from Algorithm 6.1 converge strongly to $x^{*}$ and $y^{*}$, respectively.

Remark 6.3 In view of conditions (6.2) and (6.3) (conditions (4.1) and (4.2) in [13]), we note that $k \in(0,1)$. Now, condition (6.4) (condition (4.3) in [13]) and $k>0$ imply that $2\left(r_{3}-\gamma_{3} \mu_{3}^{2}\right)<1+\mu_{3}^{2}$. Accordingly, the condition $2\left(r_{3}-\gamma_{3} \mu_{3}^{2}\right)<1+\mu_{3}^{2}$ should be added to conditions (6.2)-(6.4). On the other hand, since $k<1$, from condition (6.4) it follows that $r_{3}>\gamma_{3} \mu_{3}^{2}$.

Remark 6.4 The conditions $r_{i}>\gamma_{i} \mu_{i}^{2}+\mu_{i} \sqrt{k(2-k)}(i=1,2)$, and $k<1$ in (6.2) and (6.3) imply that $r_{i}>\gamma_{i} \mu_{i}^{2}$ for each $i=1$, 2 . Since for each $i=1,2, T_{i}$ is $\left(\gamma_{i}, r_{i}\right)$-relaxed cocoercive and $\mu_{i}$-Lipschitz continuous, the condition $r_{i}>\gamma_{i} \mu_{i}^{2}(i=1,2)$ guarantees that for each $i=1,2$, the operator $T_{i}$ is $\left(r_{i}-\gamma_{i} \mu_{i}^{2}\right)$-strongly monotone. Similarly, since $g$ is $\left(\gamma_{3}, r_{3}\right)$-relaxed cocoercive and $\mu_{3}$-Lipschitz continuous, the condition $r_{3}>\gamma_{3} \mu_{3}^{2}$ implies that the operator $g$ is $\left(r_{3}-\gamma_{3} \mu_{3}^{2}\right)$-strongly monotone.

In view of the above remarks, one can rewrite Theorem 6.2 as follows.

Theorem 6.5 Let $x^{*}, y^{*}$ be the solution of SGMVID (6.1). Let $T_{1}: \mathcal{H} \times \mathcal{H} \rightarrow \mathcal{H}$ be $\xi_{1}$-strongly monotone and $\mu_{1}$-Lipschitz continuous in the first variable, and let $T_{2}: \mathcal{H} \times$ $\mathcal{H} \rightarrow \mathcal{H}$ be $\xi_{2}$-strongly monotone and $\mu_{2}$-Lipschitz continuous in the first variable. Further, let $g$ be $\xi_{3}$-strongly monotone and $\mu_{3}$-Lipschitz continuous. If the constants $\rho$ and $\eta$ satisfy the following conditions:

$$
\left\{\begin{array}{l}
\left|\rho-\frac{\xi_{1}}{\mu_{1}^{2}}\right|<\frac{\sqrt{\xi_{1}^{2}-\mu_{1}^{2} k(2-k)}}{\mu_{1}^{2}}, \\
\left|\eta-\frac{\xi_{2}}{\mu_{2}^{2}}\right|<\frac{\sqrt{\xi_{2}^{2}-\mu_{2}^{2} k(2-k)}}{\mu_{2}^{2}}, \\
\xi_{i}>\mu_{i} \sqrt{k(2-k)}(i=1,2), \\
k=\sqrt{1-2 \xi_{3}+\mu_{3}^{2}}<1, \quad 2 \xi_{3}<1+\mu_{3}^{2},
\end{array}\right.
$$

and $\sum_{n=0}^{\infty} a_{n}=\infty$, then the iterative sequences $\left\{x_{n}\right\}$ and $\left\{y_{n}\right\}$ generated by Algorithm 6.1 converge strongly to $x^{*}$ and $y^{*}$, respectively.

\section{Conclusion}

In this paper, we have introduced and considered a new system of generalized nonlinear mixed variational inequalities involving six different nonlinear operators (SGNMVID). We have proved the equivalence between the SGNMVID and the fixed point problem, and then by this equivalent formulation, discussed the existence and uniqueness of solution of the SGNMVID. This equivalence and three nearly uniformly Lipschitzian mappings $S_{i}(i=1,2,3)$ are used to suggest and analyze some new three-step resolvent iterative schemes with mixed errors for finding an element of the set of fixed points of the nearly uniformly Lipschitzian mapping $\mathcal{Q}=\left(S_{1}, S_{2}, S_{3}\right)$, which is the unique solution of the SGNMVID. Several special cases are also considered. In Section 6, an important remark on a subclass of relaxed cocoercive mappings is discussed. It is expected that the results proved in this paper may stimulate further research regarding the numerical methods and their applications in various fields of pure and applied sciences. 


\section{Competing interests}

The authors declare that they have no competing interests.

\section{Authors' contributions}

All authors contributed equally and significantly in this paper. All authors read and approved the final manuscript.

\section{Author details}

${ }^{1}$ Department of Mathematics, Faculty of Science, Naresuan University, Phitsanulok, 65000, Thailand. ${ }^{2}$ Centre of Excellence in Mathematics, CHE, Si Ayutthaya Road, Bangkok, 10400, Thailand. ${ }^{3}$ Department of Mathematics, Sari Branch, Islamic Azad University, Sari, Iran.

\section{Acknowledgements}

The first author is supported by the Centre of Excellence in Mathematics, the commission on Higher Education, Thailand.

Received: 9 October 2012 Accepted: 19 June 2013 Published: 16 July 2013

\section{References}

1. Stampacchia, G: Formes bilineaires coercitives sur les ensembles convexes. C. R. Math. Acad. Sci. Paris 258, 4413-4416 (1964)

2. Ames, WF: Numerical Methods for Partial Differential Equations, 3rd edn. Academic Press, New York (1992)

3. Balooee, J, Cho, YJ, Kang, MK: The Wiener-Hopf equation technique for solving general nonlinear regularized nonconvex variational inequalities. Fixed Point Theory Appl. 2011, 86 (2011). doi:10.1186/1687-1812-2011-86

4. Brezis, H: Operateurs Maximaux Monotone et Semigroups de Contractions dans les Espaces de Hilbert. North-Holland, Amsterdam (1973)

5. Chang, SS, Lee, JHW, Chan, CK: Generalized system for relaxed cocoercive variational inequalities in Hilbert spaces. Appl. Math. Lett. 20, 329-334 (2007)

6. Cho, YJ, Qin, X: Systems of generalized nonlinear variational inequalities and its projection methods. Nonlinear Anal. $69,4443-4451(2008)$

7. Glowinski, R, Tallec, PL: Augmented Lagrangian and Operator-Splitting Methods in Nonlinear Mechanics. SIAM, Philadelphia (1989)

8. He, Z, Feng, G: Generalized system for relaxed cocoercive mixed variational inequalities in Hilbert spaces. Appl. Math. Comput. 214, 26-30 (2009)

9. Lions, PL, Mercier, B: Splitting algorithms for the sum of two nonlinear operators. SIAM J. Numer. Anal. 16, 964-979 (1979)

10. Liu, LS: Ishikawa and Mann iterative process with errors for nonlinear strongly accretive mappings in Banach spaces. J. Math. Anal. Appl. 194, 114-125 (1995)

11. Martinet, B: Regularization d'inequations variationelles par approximations successives. Rev. Fr. Autom. Inform. Rech. Opér. 4, 154-159 (1972)

12. Noor, MA: Splitting methods for pseudomonotone mixed variational inequalities. J. Math. Anal. Appl. 246, 174-188 (2000)

13. Noor, MA: On a system of general mixed variational inequalities. Optim. Lett. 3, 437-457 (2009)

14. Noor, MA, Huang, Z: Three-step iterative methods for nonexpansive mappings and variational inequalities. Appl. Math. Comput. 187, 680-685 (2007)

15. Peaceman, $\mathrm{DH}$, Rachford, $\mathrm{HH}$ : The numerical solution of parabolic elliptic differential equations. SIAM J. Appl. Math. 3 28-41 (1955)

16. Petrot, N: A resolvent operator technique for approximate solving of generalized system mixed variational inequality and fixed point problems. Appl. Math. Lett. 23(4), 440-445 (2010)

17. Qin, X, Cho, SY, Kang, SM: A generalized system of nonlinear variational inclusions in Hilbert spaces. Punjab Univ. J. Math. 41, 1-9 (2009)

18. Tseng, P: A modified forward-backward splitting method for maximal monotone mappings. SIAM J. Control Optim. 38, 431-446 (2000)

19. Verma, RU: Generalized system for relaxed cocoercive variational inequalities and projection methods. J. Optim. Theory Appl. 121, 203-210 (2004)

20. Verma, RU: Projection methods, algorithms, and a new system of nonlinear variational inequalities. Comput. Math Appl. 41, 1025-1031 (2001)

21. Verma, RU: General convergence analysis for two-step projection methods and applications to variational problems. Appl. Math. Lett. 18, 1286-1292 (2005)

22. Suantai, S, Petrot, N: Existence and stability of iterative algorithms for the system of nonlinear quasi-mixed equilibrium problem. Appl. Math. Lett. 24(3), 308-313 (2011)

23. Sahu, DR: Fixed points of demicontinuous nearly Lipschitzian mappings in Banach spaces. Comment. Math. Univ. Carol. 46, 653-666 (2005)

24. Rockafellar, RT: Characterization of the subdifferentials of convex functions. Pac. J. Math. 17, 497-510 (1966)

25. Chidume, CE, Chidume, CO: Convergence theorem for zeros of generalized Lipschitz generalized phi-quasi-accretive operators. Proc. Am. Math. Soc. 134, 243-251 (2005)

26. Goebel, K, Kirk, WA: A fixed point theorem for asymptotically nonexpansive mappings. Proc. Am. Math. Soc. 35 171-174 (1972)

27. Kirk, WA, Xu, HK: Asymptotic pointwise contractions. Nonlinear Anal. 69, 4706-4712 (2008)

doi:10.1186/1687-1812-2013-186

Cite this article as: Petrot and Balooee: Fixed point problems and a system of generalized nonlinear mixed variational inequalities. Fixed Point Theory and Applications 2013 2013:186. 